\title{
Least Squares Kinetic Upwind Mesh-free Method
}

\author{
S.M. Deshpande*, V. Ramesh ${ }^{\#}$, Keshav Malagi $^{\#}$, and Konark Arora ${ }^{\dagger}$ \\ "Jawaharlal Nehru Centre for Advanced Scientific Research, Bengaluru-560 064 \\ "National Aerospace Laboratories, Bengaluru-560 017 \\ 'Defence Research and Development Laboratory, Hyderabad-500 038 \\ ${ }^{*}$ E-mail: smd@jncasr.ac.in
}

\begin{abstract}
Least squares kinetic upwind mesh-free (LSKUM) method has been the subject of research over twenty years in our research group. LSKUM method requires a cloud $(\Omega)$ of points or nodes and connectivity $N\left(P_{0}\right)$ for every $P_{0} \in \Omega$. The connectivity of $P_{0}$ is a set of neighbours $P_{i} \in N\left(P_{0}\right)$ of $P_{0}$. The cloud can be a simple cloud, Cartesian cloud or chimera cloud or can be obtained rapidly using advancing front method. The discrete approximation to spatial derivatives was obtained using of least squares and it can be made accurate using defect correction method. The LSKUM first operates on the Boltzmann level and then passes on to Euler or Navier-Stokes level by taking suitable moments (so called $\psi$ moments) of the Boltzmann equation of kinetic theory of gases. The upwinding in LSKUM method is enforced by stencil or connectivity splitting based on the signs of $v_{1}, v_{2}$ in 2-D and $v_{1}, v_{2}, v_{3}$ in 3-D. This leads to split fluxes encountered in Kinetic Flux Vector Splitting (KFVS) method. The higher-order accurate LSKUM method can be made more efficient using entropy variables, thus leading to $q$-LSKUM method. Lastly, boundary conditions are implemented using specular reflection model on the wall (KCBC method) and by using kinetic outer boundary condition (KOBC) method for a point on the outer boundary.
\end{abstract}

Keywords: Boltzmann equation, kinetic theory, LSKUM method, Chimera cloud, Cartesian cloud, mesh-free method, gas dynamics, Euler equation

\section{NOMENCLATURE}

$\begin{array}{ll}A(w) & \text { Weighted least squares matrix } \\ C\left(P_{i}\right) & \text { Connectivity set } \\ E & \text { Sum of squares of deviation } \\ F & \text { Maxwellian } \\ F_{I}, F_{R} & \text { Incident/reflected Maxwellian } \\ G, G X, G Y & \text { Flux vectors } \\ G^{ \pm}, G X^{ \pm}, G Y^{ \pm} & \text {Split flux vectors } \\ N\left(P_{o}\right) & \text { Connectivity stencil / neighbourhood of } \\ & \text { a point } \\ P_{o} & \text { Node/Point under consideration } \\ q & \text { Entropy variables } \\ u, v & \text { Velocity components } \\ U & \text { Vector of conserved variables } \\ w_{i} & \text { Weights at a point } i \\ (x, y) & \text { Coordinates in global frame } \\ \left(x, y y^{\prime}\right) & \text { Coordinates in a local frame }\end{array}$

Greek Symbols

$\begin{array}{ll}\Delta \tilde{f} & \text { Modified difference } \\ \beta & \frac{1}{2 R T} \\ \rho & \text { Density }\end{array}$

Received 14 June 2010, Revised 13 July 2010

\section{INTRODUCTION}

Research work on mesh-free (grid-free or mesh-less) method began in 1989 at the CFD laboratory of the Department of Aerospace Engineering, Indian Institute of Sciences, Bangaluru ${ }^{12}$. Grid generation around complex bodies was a difficult task at that time as the commercially available grid generation packages like Gridgen, GridPro were not available then. It was therefore argued that if a cloud of point around a body can be generated quickly then a gridfree solver working on this cloud of points would be highly worthwhile. The points in the cloud are not connected by grid lines or coordinate surfaces to form tetrahedra, hexahedra or prisms; it is just a point distribution which can be generated as a Chimera cloud (Fig. 1(a), Quadtree cloud or a Cartesian cloud (Fig. 1 (b) or cloud generated by advancing front method.

It is interesting to observe that solution adaptive point enrichment is relatively easy in the grid-free frame work ${ }^{26}$. The cloud of points after point enrichment is as unstructured as the cloud before enrichment.

The last point in favour of working on grid-free method is that the clouds can be generated around a geometrically complex configuration by dividing the complexity into several geometrically simple components and then using a very fast algebraic grid generation to generate simple grids around these components. These simple grids then 

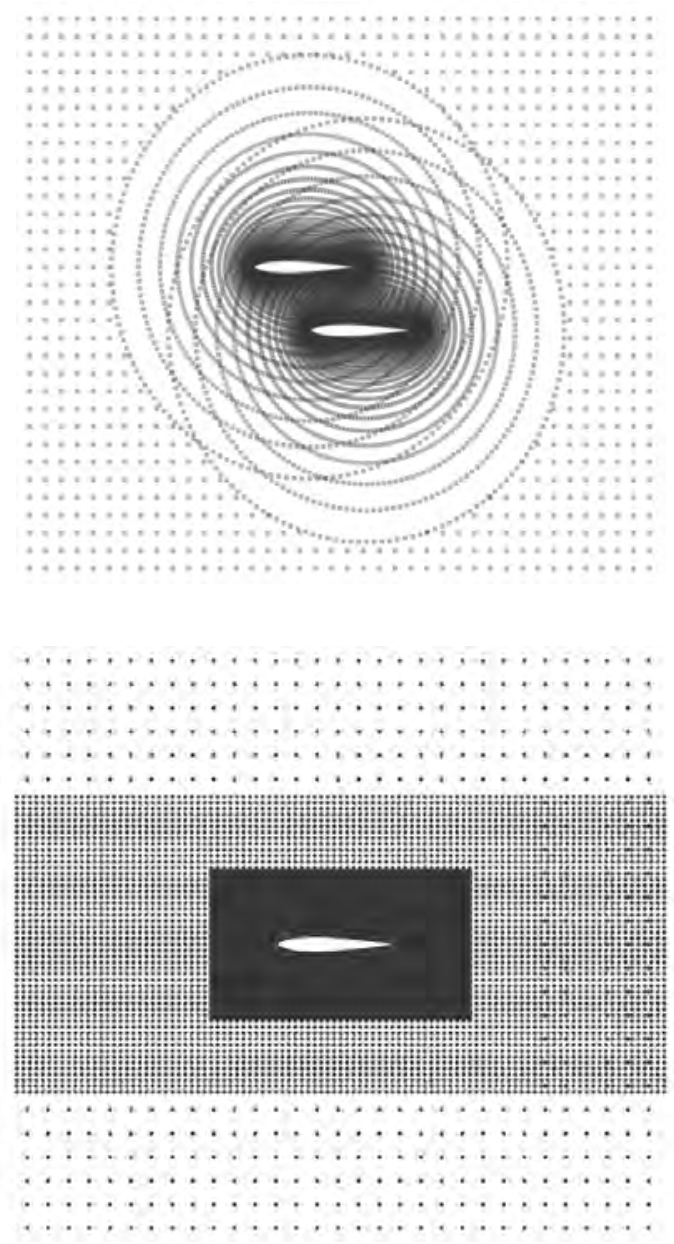

Figure 1. (a) Chimera cloud (without lines) and (b) Cartesian cloud (without lines).

overlap to form a Chimera cloud. The basic idea is to quickly generate a cloud of points and to let the grid-free solver operate on this structureless cloud to obtain numerical solution. The mesh-free method LSKUM has progressed considerably from 1989 till today and now it is a very mature tool routinely used at DRDL, Hyderabad and NAL, Bangaluru.

\section{LEAST SQUARES DISCRETISATION}

At the heart of LSKUM solver is discretisation of spatial derivates based on least squares ${ }^{15,16}$. The basic idea can be explained by taking 1-D example. Assume that derivative of $f$ wrt $x$ at node or point $x_{0}$ is required in terms of values of $f$ at neighbouring nodes $i$.

The node $P_{0}\left(x=x_{0}\right)$ has connectivity or a neighbourhood $N\left(P_{0}\right)$ consisting of points close to $P_{0}$. Referring to Fig. 2 $P_{i} \varepsilon N\left(P_{0}\right)$.

The points $P_{i} \varepsilon N\left(P_{0}\right)$ are arbitrary, these could be equally spaced or unequally spaced. There is a lot of flexibility about the choice of $N\left(P_{0}\right)$. Expanding $f_{i}$ in Taylor series around $P_{0}$ we get,

$$
\Delta f_{i}=f_{i}-f_{\circ}=\Delta x_{i} f_{x o}+\frac{\Delta x_{i}^{2}}{2} f_{x x o}+\text { H.O.T }
$$

where H.O.T= Higher-order terms. Defining the sum of squares of deviations as

$$
E=\sum_{i}\left(\Delta f_{i}-\Delta x_{i} f_{x 0}\right)^{2}, \text { where } \Delta f_{i}=f_{i}-f_{o}, \Delta x_{i}=x_{i}-x_{0}
$$

and minimising norm of $\mathrm{E}$ wrt $f_{x 0}$, one gets the first-order accurate LS (least squares) formula.

$$
f_{x 0}^{(1)}=\frac{\sum_{i} \Delta x_{i} \Delta f_{i}}{\sum_{i} \Delta x_{i}^{2}}
$$

The first-order accuracy of Eqn. (3) can be easily proved by using Taylor series Eqn. (1). Taking only 3 points in the stencil or connectivity, i.e

$$
N\left(P_{0}\right)=\left\{x_{0}, P_{1}=x_{0}+\Delta x, P_{2}=x_{0}-\Delta x\right\}
$$

the LS formula Eqn. (3) reduces to

$$
f_{x 0}^{(1)}=\frac{f_{1}-f_{2}}{2 \Delta x}
$$

which is central difference formula. If one chooses

$$
N\left(P_{0}\right)=\left\{P_{0}=x_{0}, P_{1}=x_{0}+\Delta x, P_{2}=x_{0}+2 \Delta x\right\}
$$

Then Eqn. (3) reduces to

$$
f_{x 0}^{(1)}=\frac{-3 f_{0}+f_{1}+2 f_{2}}{5 \Delta x}
$$

which is a first-order accurate forward difference formula on a 3-point stencil. For symmetric and evenly spaced points in connectivity, the LS formula in Eqn. (3) can become higher-order accurate formula. The LS formula in view of its reduction to finite difference formulae for uniform stencils, can be regarded as generalised finite differencing.

The LS formula in Eqn. (3) can be made second-order accurate by defining $E$ as

$$
E=\sum_{i}\left(\Delta f_{i}-\Delta x_{i} f_{x 0}-\frac{\Delta x_{i}^{2}}{2} f_{x x 0}\right)^{2}
$$

and then minimisation of $E$ wrt $f_{x 0}, f_{x x 0}$ yields the linear algebraic equations,

$$
\begin{aligned}
& \left(\sum \Delta x_{i}^{2}\right) f_{x o}+\left(\sum \frac{x_{i}^{3}}{2}\right) f_{x x o}=\sum \Delta f_{i} \Delta x_{i} \\
& \left(\sum \Delta \frac{x_{i}^{3}}{2}\right) f_{x o}+\left(\sum \frac{\Delta x_{i}^{4}}{4}\right) f_{x x o}=\sum \Delta f_{i} \frac{\Delta x_{i}^{2}}{2}
\end{aligned}
$$

Solving for $f_{x 0}$ we get second-order accurate formula.

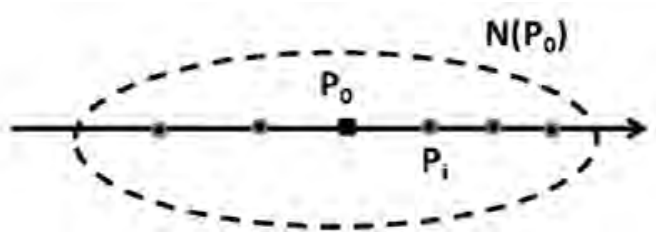

Figure 2. Connectivity of a point in one dimension. 


$$
f_{x 0}^{(2)}=\frac{\left(\Sigma \frac{\Delta x_{i}^{4}}{4}\right)\left(\Sigma \Delta f_{i} \Delta x_{i}\right)-\left(\Sigma \frac{\Delta x_{i}^{3}}{2}\right)\left(\Sigma \Delta f_{i} \frac{\Delta x_{i}^{2}}{2}\right)}{\left(\Sigma \Delta x_{i}^{2}\right)\left(\Sigma \frac{\Delta x_{i}^{4}}{4}\right)-\left(\Sigma \frac{\Delta x_{i}^{3}}{2}\right)^{2}}
$$

The denominator can become zero or close to zero in Eqn. (9), and hence, this formula can give inaccurate results for $f_{x 0}$ 'not' because derivative is ill behaved but because connectivity is bad. This method of determining spatial derivatives on arbitrary irregular grids (clouds in our terminology) has been developed by Liszka \& Orkisz ${ }^{20}$. They have also referred to problems about well conditioned FD formula. The authors have followed a different approach, namely defect correction method, for achieving higherorder accuracy of LS formulae. In the first-order formula in Eqn. (3) the denominator can never become zero. This property of Eqn. (3) was exploited and build a secondorder formula around the first-order one by using defect correction technique first employed by Ghosh \& Deshpande ${ }^{14,16}$. Multiplying Eqn. (1) by $\Delta x_{i}$ and summing over $i$ gives

$$
\Sigma \Delta f_{i} \Delta x_{i}=\left(\Sigma \Delta x_{i}^{2}\right) f_{x 0}+\left(\Sigma \frac{\Delta x_{i}^{3}}{2}\right) f_{x x 0}+\text { H.O.T }
$$

which after a slight arrangement gives

$$
f_{x 0}=\left(\frac{\Sigma \Delta f_{i} \Delta x_{i}}{\Sigma \Delta x_{i}^{2}}\right)+\left(\frac{\Sigma \frac{\Delta x_{i}^{3}}{2}}{\Sigma \Delta x_{i}^{2}}\right) f_{x x 0}+H . O . T
$$

The first term on RHS of Eqn. (10) is a first-order LS formula. For the second term

$$
\begin{aligned}
\frac{\Sigma \frac{\Delta x_{i}^{3}}{2}}{\Sigma \Delta x_{i}^{2}} f_{x x 0} & =\frac{\sum \frac{\Delta x_{i}^{2}}{2} f_{x x 0}}{\Sigma \Delta x_{i}^{2}}=\frac{\Sigma \frac{\Delta x_{i}}{2}\left(\Delta x_{i} f_{x x 0}\right)}{\Sigma \Delta x_{i}^{2}} \\
& =\frac{\sum \frac{\Delta x_{i}}{2}\left(f_{x i}-f_{x 0}\right)}{\Sigma \Delta x_{i}^{2}}+\text { H.O.T }
\end{aligned}
$$

Substitution in Eqn. (10) yields

$$
f_{x 0}=\frac{\Sigma \Delta x_{i}\left[\Delta f_{i}-\frac{\Delta x_{i}}{2}\left(f_{x i}-f_{x 0}\right)\right]}{\Sigma \Delta x_{i}^{2}}
$$

Defining the modified difference

$$
\begin{aligned}
\Delta \tilde{f}_{i} & =\Delta f_{i}-\frac{\Delta x_{i}}{2}\left(f_{x i}-f_{x o}\right) \\
& =\left(f_{i}-\frac{\Delta x_{i}}{2} f_{x i}\right)-\left(f_{0}-\frac{\Delta x_{i}}{2} f_{x 0}\right)
\end{aligned}
$$

We then get the second-order accurate LS formula

$$
f_{x 0}=\frac{\Sigma x_{i} \Delta \tilde{f}_{i}}{\Sigma \Delta x_{i}^{2}}
$$

which has the same non-zero positive denominator as in the first-order accurate LS formula [Eqn. (3)]. Several comments on Eqn. (13) are in order now.

(i) It has the same structure as the first-order LS formula Eqn. (3), modified or perturbed difference Eqn. (12) appears in Eqn. (13) instead of $\Delta f_{i}$ in Eqn. (3).

(ii) The formula in Eqn. (13) is implicit in the sense that value of derivative at node $P_{0}$ depends on its values of derivatives at other nodes.

(iii) Due to its implicit nature the formula in Eqn. (13) has a better spectral bandwidth for scale resolution. To demonstrate this property, a stencil as shown in Fig. 3 was chosen.

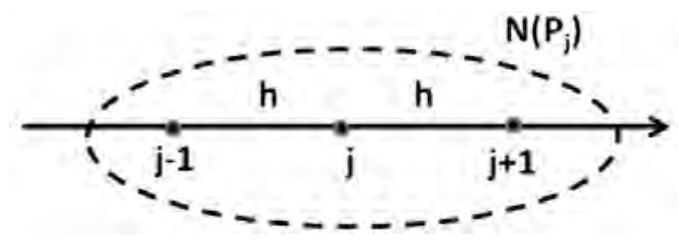

Figure 3. Stencil of a point in one dimension.

The point $P_{i}$ has evenly spaced 3-Point stencil. The LS formula in Eqn. (13) then reduces to

$$
\begin{gathered}
f_{x j}=\frac{\left[\begin{array}{l}
-h\left(f_{j-1}-f_{j}\right)+h\left(f_{j+1}-f_{j}\right) \\
-\frac{h^{2}}{2}\left(f_{x_{j-1}}-f_{x j}\right)-\frac{h^{2}}{2}\left(f_{x_{j+1}}-f_{x j}\right)
\end{array}\right]}{\left(2 h^{2}\right)} \\
=\frac{f_{j+1}-f_{j-1}}{2 h}-\left(\frac{1}{4} \Sigma f_{x_{j-1}}-\frac{1}{2} f_{x j}+\frac{1}{4} f_{x_{j+1}}\right)
\end{gathered}
$$

Or equivalently

$$
\frac{1}{4} f_{x j-1}+\frac{1}{2} f_{x j}+\frac{1}{4} f_{x j+1}=\frac{f_{j+1}-f_{j-1}}{2 h}
$$

The LS formula in Eqn. (14) is one special case of compact difference formulae due to Lele $^{21}$ and these are known to have better spectral resolution property.

(iv) Iterations or some kind of inner iterations are required to obtain higher accurate values of $f_{x o}$ from Eqn. (13). One possible of cycle of iterations could be

$$
\left.\begin{array}{l}
f_{x 0}^{(1)}=\frac{\Sigma \Delta x_{i} \Delta f_{i}}{\Sigma \Delta x_{i}^{2}} \\
\left.\tilde{f}_{x 0}=\frac{\Sigma \Delta x_{i}\left[\Delta f_{i}-\frac{\Delta x_{i}}{2}\left(f_{x i}^{(1)}-f_{x 0}^{(1)}\right)\right]}{\Sigma \Delta x_{i}^{2}}\right] \\
\left.\tilde{\tilde{f}}_{x 0}=\frac{\Sigma \Delta x_{i}\left[\Delta f_{i}-\frac{\Delta x_{i}}{2}\left(\tilde{f}_{x i}-\tilde{f}_{x 0}\right)\right]}{\Sigma \Delta x_{i}^{2}}\right]
\end{array}\right\}
$$


Let us get back to first-order LS formula in Eqn. (3) for spatial derivative $f_{\mathrm{x} 0}$. It is possible to use weighted least squares. Introducing weights in Eqn. (2) we get,

$$
E=\sum_{i} w_{i}\left(\Delta f_{i}-\Delta x_{i} f_{x o}\right)^{2}, \quad \Delta x_{i}=x_{i}-x_{0}, \quad \Delta f_{i}=f_{i}-f_{0}
$$

Minimising E wrt. $f_{x o}$ one gets weighted LS formula

$$
\left.f_{x 0}^{(1)}=\frac{\sum w_{i} \Delta x_{i} \Delta f_{i}}{\sum w_{i} \Delta x_{i}^{2}}\right\}
$$

and the denominator will be positive if all $w_{i}>0$. The formula in Eqn. (16) is a weighted least squares formula for derivative $f_{\mathrm{x} 0}$. The truncation error in Eqn. (16) is given by

$$
\mathrm{T}_{r}=\left(\frac{\Sigma w_{i} \frac{\Delta x_{i}^{3}}{2}}{\Sigma w_{i} \Delta x_{i}^{2}}\right) f_{x x o}=O\left(\Delta x_{\max }\right), \Delta x_{\max }=\max _{i}\left\{\Delta x_{i}\right\}
$$

There are several ways in which weights $w_{i}$ can be determined. One possibility is to minimise the coefficient of $f_{x x 0}$ in Eqn. (17) thereby making the formula in Eqn. (16) more accurate. Yet another possibility is to choose

$$
w_{i}=\left(\frac{\Delta x_{m}}{\Delta x_{i}}\right)^{r}
$$

where $\Delta x_{m}=\min _{i}\left\{\Delta x_{i}\right\}, r$ is any positive integer. The idea in choosing Eqn. (18) is to reduce weight attached to points far away from the node $P_{0}$ and thus make the weighted LS formula more local. Yet another possibility is to choose weights for increasing the spectral bandwidth of Eqn. (16). To demonstrate the ideas behind spectral bandwidth, consider the choice

$$
f(x)=e^{i k x}
$$

Which gives $f_{x 0}^{\text {exact }}=\left(e^{i k x_{0}}\right) i k$

Substituting for $f(x)$ in Eqn. (16) one gets

$$
f_{x 0}^{(1)}=\frac{\sum w_{j} \Delta x_{j}\left(e^{i k x_{j}}-e^{i k x_{0}}\right)}{\sum w_{j} \Delta x_{j}^{2}}
$$

We can easily verify that

$$
f_{x 0}^{(1)}=i k e^{i k x_{0}}-\left(\frac{\sum w_{j} \frac{\Delta x_{j}^{3}}{2} k^{2}}{\Sigma w_{j} \Delta x_{j}^{2}}\right)+\text { H.O.T }
$$

Noting that

$$
\left|f_{x 0}^{(1)}-f_{x 0}^{\text {exact }}\right|^{2}=\left|\left(\frac{\sum w_{j} \Delta x_{j}\left(e^{i k \Delta x_{j-1}}\right)}{\Sigma w_{j} \Delta x_{j}^{2}}\right)-i k\right|^{2}
$$

One can choose weights so as to minimise a norm

$$
E=\int_{k_{0}}^{k_{1}}\left|f_{x 0}^{(1)}-f_{x 0}^{\text {exact }}\right|^{2} d k
$$

which is an integral of the square of the difference over the bandwidth $k_{0} \leq k \leq k_{1}$. Alternatively choose weights $w_{j}$ such that

$$
\left(\Sigma w_{j} \frac{\Delta x_{j}^{3}}{2}\right)=0
$$

So as to make $f_{x 0}^{(1)}$ second-order accurate.

\section{MULTI-DIMENSIONAL LEAST SQUARE FORMULA}

Taking $f(x, y)$ to be two-dimensional functions, we define

$$
E=\sum_{i} w_{i}\left(\Delta f_{i}-\Delta x_{i} f_{x 0}-\Delta y_{i} f_{y o}\right)^{2}
$$

Here $N\left(P_{0}\right)$ is the connectivity of node $P_{0}\left(x_{0}, y_{0}\right)$ (Fig. 4) at which we want to derive LS formulae for the derivatives $f_{x 0}, f_{y 0}$. Minimising $E$ wrt $f_{x 0}, f_{y 0}$ we obtain

$$
\left.\begin{array}{l}
\left(\Sigma w_{i} \Delta x_{i}^{2}\right) f_{x 0}+\left(\Sigma w_{i} \Delta x_{i} \Delta y_{i}\right) f_{y 0}=\Sigma w_{i} \Delta x_{i} \Delta f_{i} \\
\left(\Sigma w_{i} \Delta x_{i} \Delta y_{i}\right) f_{x 0}+\left(\Sigma w_{i} \Delta y_{i}^{2}\right) f_{y 0}=\Sigma w_{i} \Delta y_{i} \Delta f_{i}
\end{array}\right\}
$$

The LS matrix $A(w)$ defined by

$$
A(w)=\left[\begin{array}{cc}
\Sigma w_{i} \Delta x_{i}^{2} & \Sigma w_{i} \Delta x_{i} \Delta y_{i} \\
\Sigma w_{i} \Delta x_{i} \Delta y_{i} & \Sigma w_{i} \Delta y_{i}^{2}
\end{array}\right]
$$

is a $2 \times 2$ symmetric matrix and has

$$
\operatorname{det}[A(w)]=\left(\sum w_{i} \Delta x_{i}^{2}\right)\left(\sum w_{i} \Delta y_{i}^{2}\right)-\left(\sum w_{i} \Delta x_{i} \Delta y_{i}\right)^{2}
$$

The determinant is always non-zero due to CauchySchwartz inequality. The $\operatorname{det}[A(w)]$ will be zero if all the

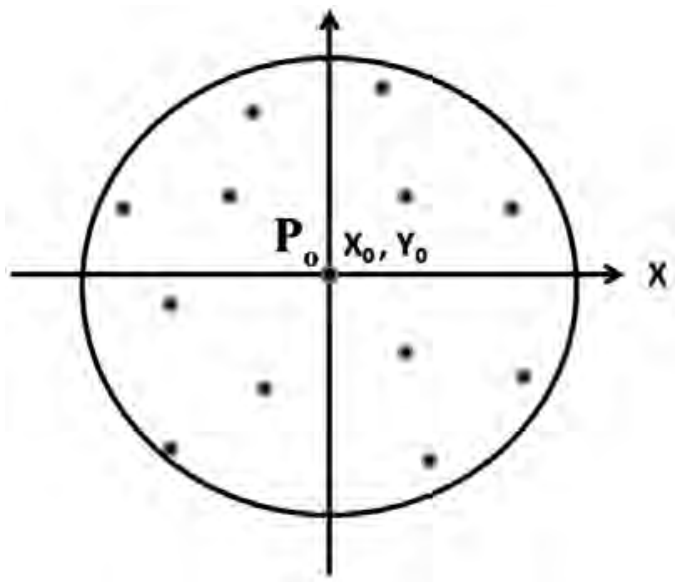

Figure 4. Connectivity of node $P_{0}$ in two dimensions. 
points $x_{i}, y_{i}$ fall on a straightline in which case it will not be 2-D distribution of points. Solving Eqn. (26) for $f_{x 0}, f_{y 0}$ we get

$$
\begin{aligned}
& f_{x 0}=\frac{\sum \Delta y_{i}^{2} \sum \Delta x_{i} \Delta f_{i}-\sum \Delta x_{i} \Delta y_{i} \sum \Delta y_{i} \Delta f_{i}}{\operatorname{det}[A(w)]} \\
& \left.f_{y 0}=\frac{\sum \Delta x_{i}^{2} \sum \Delta y_{i} \Delta f_{i}-\sum \Delta x_{i} \Delta y_{i} \sum \Delta x_{i} \Delta f_{i}}{\operatorname{det}[A(w)]}\right\}
\end{aligned}
$$

Again several observations can be made. Let the coordinate system $x, y$ be rotated to $x^{\prime}, y^{\prime}$. The LS formulae for derivatives in $x^{\prime}, y^{\prime}$ frame are easily obtained by changing $x, y$ into $x^{\prime}, y^{\prime}$ in Eqn. (29). This invariance of form of formulae (or covariant formulae) follows from Eqn. (25) which in the transformed frame becomes

$$
E=\sum_{i} w_{i}\left(\Delta f_{i}-\Delta x_{i}^{\prime} f_{x^{\prime} 0}-\Delta y_{i}^{\prime} f_{y^{\prime} o}\right)^{2}
$$

The rest of derivation for $f_{x^{\prime} 0}, f_{y^{\prime} o}$ then easily follows. The invariance of the inner product ${ }^{14,24}$

$$
\Delta x_{i} f_{x 0}+\Delta y_{i} f_{y 0}
$$

between gradient vector $f_{x 0}, f_{y 0}$ and coordinate differentials vector carries over to invariance of

$$
\Delta x_{i} f_{x 0}^{(1)}+\Delta y_{i} f_{y 0}^{(1)}
$$

in which discrete LS approximation Eqn. (29) is employed. Assuming $v_{1}, v_{2}$ to be particle velocity vector (commonly used in construction of Boltzmann schemes) one could similarly conclude that,

$$
v_{1} f_{x 0}^{(1)}+v_{2} f_{y 0}^{(1)}
$$

will also be invariant under rotation. $\mathrm{Keshav}^{24}$ and Keshav ${ }^{25}$, et al. have exploited this property in constructing rotationally invariant LSKUM called KUMARI for inviscid gas dynamics.

The rotation of global frame $x, y$ to local frame $x$, $y^{\prime}$ is useful in application of LSKUM to problems involving flow past bodies.

A point $P_{0}$ is on the solid boundary (Fig. 5). Here $x, y$ in the global frame and $\left(x^{\prime}, y^{\prime}\right)$ is the locally rotated frame so that $x^{\prime}$ is along the body and $y^{\prime}$ normal to it. The flow tangency gives

$$
\vec{u} \cdot \hat{n}\left(P_{0}\right)=0
$$

where, $\vec{u}=$ fluid velocity vector and $\hat{n}\left(P_{0}\right)$ is the unit normal vector at $P_{0}$ It is easier to enforce Eqn. (34) in the local frame $\left(x^{\prime}, y^{\prime}\right)$. At an interior point also sometimes it is profitable to use $x^{\prime}, y^{\prime}$ with $x^{\prime}$ along the streamline and $y$ ' normal to it. Dauhoo ${ }^{8}$ has made use of LSKUM with streamline upwinding by rotating coordinate frame.

Yet another observation about the LS formulae Eqn. (29) is that onee can choose weights $w_{i}$ in such a way that the LS matrix $A(w)$ becomes a diagonal matrix, that is, choose $w_{i}$ to satisfy

$$
\Sigma w_{i} \Delta x_{i} \Delta y_{i}=0
$$

The LS formulae Eqn. (29) then reduce to 1-D like formulae. Konark and Deshpande ${ }^{4}$ have developed a method to determine weights $w_{i}$ to satisfy Eqn. (35). Denoting these weights $\tilde{w}_{i}^{*}$ by the multi-dimensional LS formula for spatial derivatives reduce to $1-\mathrm{D}$ LS formula

$$
f_{x o}=\frac{\sum \tilde{w}_{i}^{*} \Delta f_{i} \Delta x_{i}}{\sum \tilde{w}_{i}^{*} \Delta x_{i}^{2}}, f_{y o}=\frac{\sum \tilde{w}_{i}^{*} \Delta f_{i} \Delta y_{i}}{\sum \tilde{w}_{i}^{*} \Delta y_{i}^{2}}
$$

The weights $w_{i}^{*}, \tilde{w}_{i}^{*}$ used in formulae for $f_{x o}$ and $f_{y o}$ are generally different ${ }^{5-7}$. Here, weights $w_{i}^{*}, \tilde{w}_{i}^{*}$ are the weights for the diagonalisation of $A(w)$. One of the advantages of 1-D like formula Eqn. (36) apart from its simplicity is the fact that denominators in Eqn. (36) can never be zero if weights $w_{i}^{*}$ are positive. The rank deficiency problem of $A(w)$ can therefore be tackled by use of 1-D formulae Eqn. (36). Arora ${ }^{6}$, Arora, Rajan and Deshpande ${ }^{7}$ and Arora and Deshpande ${ }^{4}$ have used the above diagonalisation in construction of their robust and accurate LSKUM.

The order of accuracy of multi-dimensional LS formulae can be enhanced using the defect correction technique ${ }^{14-16}$ explained in the previous section. For this purpose they first write Eqn. (26) in the matrix form

$$
A(w)\left[\begin{array}{l}
f_{x o} \\
f_{y o}
\end{array}\right]=\left[\begin{array}{l}
\sum w_{i} \Delta x_{i} \Delta f_{i} \\
\sum w_{i} \Delta y_{i} \Delta f_{i}
\end{array}\right]
$$

write $E$ defined by Eqn. (25) as

$$
E=\sum_{i} w_{i}\left(\begin{array}{l}
\Delta f_{i}-\Delta x_{i} f_{x o}-\Delta y_{i} f_{y o}-\frac{\Delta x_{i}^{2}}{2} f_{x x o} \\
-\Delta x_{i} \Delta y_{i} f_{x y o}-\frac{\Delta y_{i}^{2}}{2} f_{y y o}
\end{array}\right)^{2}
$$

For the purpose of deriving second-order accurate LS formulae. Again minimising $E$ wrt. $f_{x o}, f_{y o}$ one gets

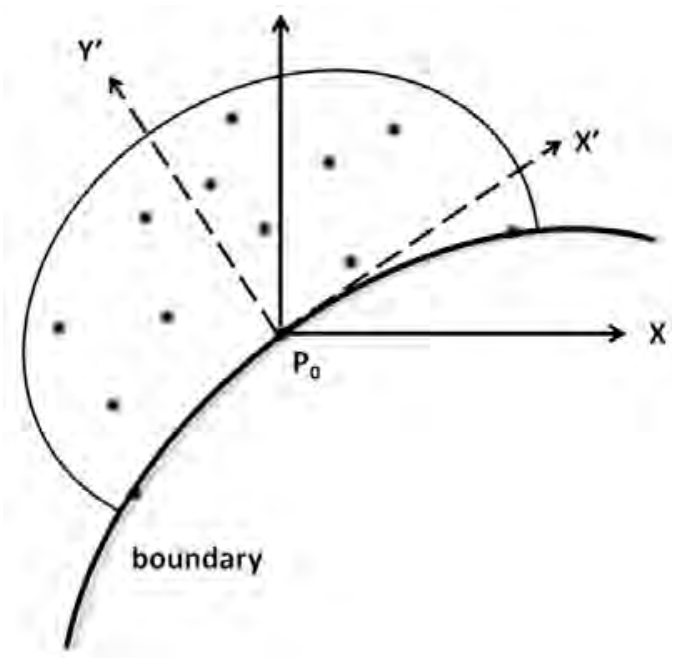

Figure 5. Point distribution around a boundary point $\boldsymbol{P}_{0}$ 


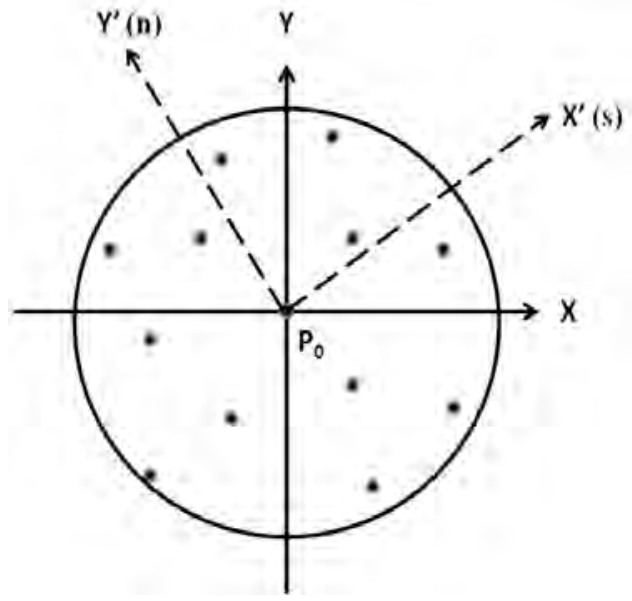

Figure 6. Point distribution around an interior point $\boldsymbol{P}_{0}$.

$$
\begin{aligned}
& \left(\sum w_{i} \Delta x_{i}^{2}\right) f_{x o}+\left(\sum w_{i} \Delta x_{i} \Delta y_{i}\right) f_{y o}=R H S_{1} \\
& =\left(\sum w_{i} \Delta x_{i} \Delta f_{i}\right)-\sum w_{i} \Delta x_{i}\left\{\frac{\Delta x_{i}^{2}}{2} f_{x x o}+\Delta x_{i} \Delta y_{i} f_{x y o}+\frac{\Delta y_{i}^{2}}{2} f_{y y o}\right\} \\
& \left(\sum w_{i} \Delta x_{i} \Delta y_{i}\right) f_{x o}+\left(\sum w_{i} \Delta y_{i}^{2}\right) f_{y o}=R H S_{2} \\
& =\left(\sum w_{i} \Delta y_{i} \Delta f_{i}\right)-\sum w_{i} \Delta y_{i}\left\{\frac{\Delta x_{i}^{2}}{2} f_{x x o}+\Delta x_{i} \Delta y_{i} f_{x y o}+\frac{\Delta y_{i}^{2}}{2} f_{y y o}\right\}
\end{aligned}
$$

One wants to prove that

$$
\frac{\Delta x_{i}^{2}}{2} f_{x x o}+\Delta x_{i} \Delta y_{i} f_{x y o}+\frac{\Delta y_{i}^{2}}{2} f_{y y o}=\frac{1}{2} \Delta x_{i} \Delta f_{x i}+\frac{1}{2} \Delta y_{i} \Delta f_{y i}+H . O T .
$$

where $\Delta f_{x i}=f_{x i}-f_{x o} ; \Delta f_{y i}=f_{y i}-f_{y o}$

One may note that

$$
\begin{aligned}
& \Delta f_{x i}=f_{x i}-f_{x o}=f_{x x o} \Delta x_{i}+f_{x y o} \Delta y_{i}+\text { H.O.T. } \\
& \Delta f_{x i}=f_{x i}-f_{x o}=f_{x y o} \Delta x_{i}+f_{y y o} \Delta y_{i}+\text { H.O.T. }
\end{aligned}
$$

and therefore we get

$$
\frac{1}{2} \Delta x_{i} \Delta f_{x i}+\frac{1}{2} \Delta y_{i} \Delta f_{y i}=\frac{\Delta x_{i}^{2}}{2} f_{x x o}+\Delta x_{i} \Delta y_{i} f_{x y o}+\frac{\Delta y_{i}^{2}}{2} f_{y y o}+\text { H.O.T. }
$$

which is identical with Eqn. (40). The RHS of Eqn. (39) is therefore given by

$$
\begin{aligned}
R H S_{1} & =\sum w_{i} \Delta x_{i}\left\{\Delta f_{i}-\frac{\Delta x_{i}}{2} \Delta f_{x i}-\frac{\Delta y_{i}}{2} \Delta f_{y i}\right\} \\
& =\sum w_{i} \Delta x_{i}\left\{\left(f_{i}-\frac{\Delta x_{i}}{2} f_{x i}-\frac{\Delta y_{i}}{2} f_{y i}\right)-\left(f_{o}-\frac{\Delta x_{i}}{2} f_{x o}-\frac{\Delta y_{i}}{2} f_{y o}\right)\right\} \\
& =\sum w_{i} \Delta x_{i} \Delta \tilde{f}_{i}
\end{aligned}
$$

Similarly,

$$
\begin{aligned}
R H S_{2} & =\sum w_{i} \Delta y_{i}\left\{\Delta f_{i}-\frac{\Delta x_{i}}{2} \Delta f_{x i}-\frac{\Delta y_{i}}{2} \Delta f_{y i}\right\} \\
& =\sum w_{i} \Delta y_{i} \Delta \tilde{f}_{i}
\end{aligned}
$$

The second-order accurate LS formulae therefore take on the first-order form

$$
A(w)\left[\begin{array}{l}
f_{x o} \\
f_{y o}
\end{array}\right]=\left[\begin{array}{l}
\sum w_{i} \Delta x_{i} \Delta \tilde{f}_{i} \\
\sum w_{i} \Delta y_{i} \Delta \tilde{f}_{i}
\end{array}\right]
$$

We note that the modified difference $\Delta \widetilde{f}_{i}$

$$
\begin{aligned}
\Delta \tilde{f}_{i} & =\Delta f_{i}-\frac{\Delta x_{i}}{2} \Delta f_{x i}-\frac{\Delta y_{i}}{2} \Delta f_{y i} \\
& =\left(f_{i}-f_{o}\right)-\frac{\Delta x_{i}}{2}\left(f_{x i}-f_{x o}\right)-\frac{\Delta y_{i}}{2}\left(f_{y i}-f_{y o}\right) \\
& =\left(f_{i}-\frac{\Delta x_{i}}{2} f_{x i}-\frac{\Delta y_{i}}{2} f_{y i}\right)-\left(f_{o}-\frac{\Delta x_{i}}{2} f_{x o}-\frac{\Delta y_{i}}{2} f_{y o}\right) \\
& =\tilde{f}_{i}-\tilde{f}_{o}
\end{aligned}
$$

where modified $f$ at node $i$ is

$$
\tilde{f}_{i}=f_{i}-\frac{\Delta x_{i}}{2} f_{x i}-\frac{\Delta y_{i}}{2} f_{y i}
$$

and modified $f$ at node $o$ is

$$
\tilde{f}_{o}=f_{o}-\frac{\Delta x_{i}}{2} f_{x o}-\frac{\Delta y_{i}}{2} f_{y o}
$$

In obtaining modified $f$, the midpoint $\left(\frac{\Delta x_{i}}{2}, \frac{\Delta y_{i}}{2}\right)$ (Fig. 7) was used. Modified $\tilde{f}_{i}, \tilde{f}_{o}$ use derivatives at nodes $o$ and $i$ and these spatial derivatives were used to obtain $\tilde{f}_{i}, \tilde{f}_{o}$. The defect correction technique for second-order accuracy has been used by Anandhanarayanan ${ }^{2}$, Arora $^{6}$, Dauhoo $^{8}$, Ghosh $^{16}$, Praveen ${ }^{27}$ and Ramesh ${ }^{29}$.

The extension of second-order accurate LS formula Eqn. (44) to 3-D is now obvious. We write by inspection the 3-D formulae

$$
A(w)\left[\begin{array}{c}
f_{x o} \\
f_{y o} \\
f_{z o}
\end{array}\right]=\left[\begin{array}{l}
\sum w_{i} \Delta x_{i} \Delta \tilde{f i} \\
\sum w_{i} \Delta y_{i} \Delta \tilde{f}_{i} \\
\sum w_{i} \Delta z_{i} \Delta \tilde{f}_{i}
\end{array}\right]
$$

where

$$
\Delta \tilde{f}_{i}=\Delta f_{i}-\frac{\Delta x_{i}}{2} \Delta f_{x i}-\frac{\Delta y_{i}}{2} \Delta f_{y i}-\frac{\Delta z_{i}}{2} \Delta f_{z i}
$$

As mentioned before, the above LS formulae Eqn. (44) and Eqn. (48) are implicit in nature as the RHS depends upon spatial derivatives at nodes $o$ and $i$. A few iterations are therefore required to compute $f_{x o}, f_{y o}, f_{z o}$ at node $o$ to second-order accuracy.

\section{LSKUM-FREE FOR EULER EQUATIONS OF GAS DYNAMICS}

The LS approximations to spatial derivatives was considered in terms of data at nodes in the connectivity. These ideas were applied to develop Least Squares Kinetic upwind mesh-free (LSKUM) method for obtaining numerical solution of Euler equations of gas dynamics. We illustrate the basic principles behind LSKUM method by considering 


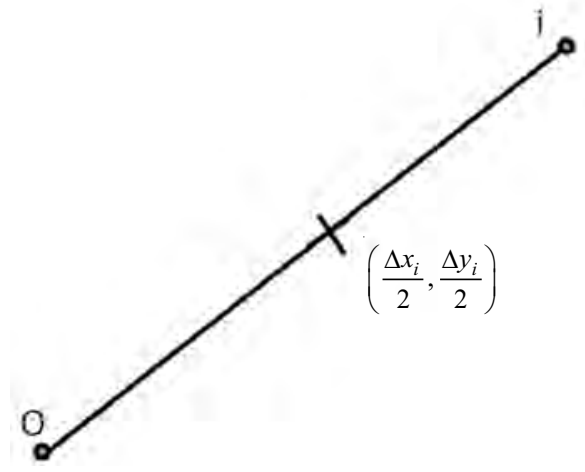

Figure 7. Midpoint used in evaluating modified $f(\tilde{f})$.

1-D problem. Also, moment method strategy ${ }^{11}$ is used. Here the Euler equations are obtained as suitable moments of the Boltzmann equation of kinetic theory of gases. It may be noted that Finite Pointset mesh-free method for incompressible viscous flows employing weighted least squares approach has been developed by Tiwari and Kunhert ${ }^{32}$ and Kunhert ${ }^{18}$ have developed upwind finite pointset method (FPM) for compressible Euler and Navier-Stokes equations. They have on the other hand developed kinetic mesh-free method and used defect correction technique for higherorder accuracy.

For 1-D problem, the Boltzmann equation without collision term is given by

$$
\frac{\partial F}{\partial t}+v \frac{\partial F}{\partial x}=0
$$

where $F$ is the Maxwellian velocity distribution defined by

$$
F=\frac{\rho}{I_{o}} \sqrt{\frac{\beta}{\pi}} \exp \left(-\beta(v-u)^{2}-\frac{I}{I_{o}}\right)
$$

Here $\rho$ is the mass density, $\beta=1 /(2 R T), R=$ gas constant, $T=$ absolute temperature, $u=$ fluid velocity and $v=$ particle or molecular velocity, $I_{o}=$ average internal energy due to non translational degrees of freedom $=\frac{2+D-\gamma D}{2(\gamma-1)} R T, D=$ degrees of freedom, The $\psi-$ moments ${ }^{11,22}$ yields 1 -D Euler equations

$$
\frac{\partial U}{\partial t}+\frac{\partial G}{\partial x}=0
$$

where $U=\int_{0}^{\infty} d I \int_{-\infty}^{+\infty} \psi F d v, G=\int_{0}^{\infty} d I \int_{-\infty}^{+\infty} v \psi F d v$ and

$$
\psi=\left[\begin{array}{c}
1 \\
v \\
I+\frac{v^{2}}{2}
\end{array}\right]
$$

It is noted that $U$ and $G$ are vectors given by

$$
U=\left[\begin{array}{c}
\rho \\
\rho u \\
e
\end{array}\right], G=\left[\begin{array}{c}
\rho u \\
p+\rho u^{2} \\
(e+p) u
\end{array}\right]
$$

where $p=$ pressure, $e=$ total energy per unit volume $=\frac{p}{(\gamma-1)}+\frac{1}{2} \rho u^{2}$. Kinetic flux-vector splitting ${ }^{22}$ is obtained by using Courant splitting, that is write Eqn. (50) as

$$
\frac{\partial F}{\partial t}+\frac{v+|v|}{2} \frac{\partial F}{\partial x}+\frac{v-|v|}{2} \frac{\partial F}{\partial x}=0
$$

Taking $\psi-$ moments of Eqn. (55), one gets KFVS (Kinetic Flux Vector Split) ${ }^{22,23}$ Euler equations.

$$
\frac{\partial U}{\partial t}+\frac{\partial G^{+}}{\partial x}+\frac{\partial G^{-}}{\partial x}=0
$$

where $G^{ \pm}=\int_{0}^{\infty} d I \int \frac{v \pm|v|}{2} \psi F d v$ and further

$$
G^{+}=\left[\begin{array}{c}
\rho u A^{+}+\rho B \\
\left(p+\rho u^{2}\right) A^{+}+\rho u B \\
(e+p) u A^{+}+\left(e+\frac{p}{2}\right) B
\end{array}\right], G^{-}=\left[\begin{array}{c}
\rho u A^{-}-\rho B \\
\left(p+\rho u^{2}\right) A^{-}-\rho u B \\
(e+p) u A^{-}-\left(e+\frac{p}{2}\right) B
\end{array}\right]
$$

where $A^{ \pm}=\frac{1 \pm \operatorname{erf}(S)}{2}, B=\frac{e^{-S^{2}}}{2 \sqrt{\beta \pi}}, S=u \sqrt{\beta}$

With this background material, they are ready to develop LSKUM $^{15,16}$ for the 1-D Euler equation Eqn. (52). In the Courant split Eqn. (55), we replaced the space derivatives $\frac{\partial F}{\partial x}$ by the LS approximations, and then by taking $\psi-$ moments we obtain.

$$
\left(\frac{\partial U}{\partial t}\right)_{o}^{n}+\left(\frac{\sum_{i} \Delta G_{i}^{+} \Delta x_{i}}{\sum_{i} \Delta x_{i}^{2}}\right)_{i \in C^{+}\left(P_{o}\right)}+\left(\frac{\sum_{i} \Delta G_{i}^{-} \Delta x_{i}}{\sum_{i} \Delta x_{i}^{2}}\right)_{i \in C^{-}\left(P_{o}\right)}=0
$$

The notation used in Eqn. (58) needs explanation. The connectivity $C^{+}\left(P_{o}\right)$ is of node $P_{o}$ and it is split into $C^{+}\left(P_{o}\right)$ and $C^{-}\left(P_{o}\right)$ which are defined by

$$
\begin{aligned}
& C^{+}\left(P_{o}\right)=\left\{\forall i \mid x_{i}-x_{o} \leq 0\right\} \\
& C^{-}\left(P_{o}\right)=\left\{\forall i \mid x_{i}-x_{o} \geq 0\right\}
\end{aligned}
$$

The split connectivity $C^{+}\left(P_{o}\right)$ is used in discretising the term

$$
\frac{v+|v|}{2} \frac{\partial F}{\partial x}
$$


and split connectivity $C^{-}\left(P_{o}\right)$ is used in discretising the term

$$
\frac{v-|v|}{2} \frac{\partial F}{\partial x}
$$

The suffix $i$ runs through $C^{+}\left(P_{d}\right)$ in the LS expression for discretising Eqn. (60) and it runs through $C^{-}\left(P_{o}\right)$ for discretising Eqn. (61). Using first-order time marching for the time derivative in Eqn. (58) we get the LSKUM methodbased state update.

$$
U^{n+1}\left(P_{o}\right)=U^{n}\left(P_{o}\right)-\Delta t\left(\frac{\sum_{i} \Delta G_{i}^{+} \Delta x_{i}}{\sum \Delta x_{i}^{2}}\right)_{i \in C^{+}\left(P_{o}\right)}-\Delta t\left(\frac{\sum_{i} \Delta G_{i}^{-} \Delta x_{i}}{\sum \Delta x_{i}^{2}}\right)_{i \in C^{-}\left(P_{o}\right)}
$$

For the sake of easy understanding Eqn. (62) we repeat the definitions

$$
\Delta G_{i}^{ \pm}=G_{i}^{ \pm}-G_{o}^{ \pm}, \Delta x_{i}=x_{i}-x_{o}
$$

The LSKUM-based state update Eqn. (62) uses the KFVS fluxes $G^{ \pm}$and stencil splitting for obtaining an upwind numerical scheme at Euler level. We do velocity splitting at the Boltzmann level and then pass on to the Euler level by taking $\psi$-moments of the split Boltzmann Eqn. (55). The state update Eqn. (62) is first-order accurate in space and time. The second-order accuracy in time can be easily obtained by using RK-2/RK-4 in Eqn. (58). The second-order accuracy in space can be achieved using defect correction. Using the theory explained earlier in Section 3 , we defined modified flux differences $\Delta \tilde{G}^{ \pm}$by

$$
\begin{aligned}
& \Delta \tilde{G}_{i}^{ \pm}=\Delta G_{i}^{ \pm}-\frac{\Delta x_{i}}{2} \Delta\left(\frac{\partial G^{ \pm}}{\partial x}\right)_{i} \\
& =\left(G_{i}^{ \pm}-G_{o}^{ \pm}\right)-\frac{\Delta x_{i}}{2}\left[\left(\frac{\partial G^{ \pm}}{\partial x}\right)_{i}-\left(\frac{\partial G^{ \pm}}{\partial x}\right)_{o}\right] \\
& =\left\{G_{i}^{ \pm}-\frac{\Delta x_{i}}{2}\left(\frac{\partial G^{ \pm}}{\partial x}\right)\right\}-\left\{G_{o}^{ \pm}-\frac{\Delta x_{i}}{2}\left(\frac{\partial G^{ \pm}}{\partial x}\right)_{o}\right\}
\end{aligned}
$$

The second-order accurate LS formulae for space derivative then yields the semi discrete law

$$
\left(\frac{\partial U}{\partial t}\right)_{o}^{n}+\left(\frac{\sum_{i} \Delta \tilde{G}_{i}^{+} \Delta x_{i}}{\sum \Delta x_{i}^{2}}\right)_{i \in C^{+}\left(P_{o}\right)}+\left(\frac{\sum_{i} \Delta \tilde{G}_{i}^{-} \Delta x_{i}}{\sum \Delta x_{i}^{2}}\right)_{i \in C^{-}\left(P_{o}\right)}=0
$$

We can use RK2 or RK4 for higher-order accuracy in time. It may be noted that as mentioned before a few inner iterations are generally required to achieve secondorder accuracy for space derivatives. The extension of Eqn. (65) to multidimensional flows is straight forward. We have to discretise the 2-D Euler equation

$$
\frac{\partial U}{\partial t}+\frac{\partial}{\partial x}(G X)+\frac{\partial}{\partial y}(G Y)=0
$$

Here

$$
U=\left[\begin{array}{c}
\rho \\
\rho u_{1} \\
\rho u_{2} \\
e
\end{array}\right], G X=\left[\begin{array}{c}
\rho u_{1} \\
p+\rho u_{1}^{2} \\
\rho u_{1} u_{2} \\
(e+p) u_{1}
\end{array}\right], G Y=\left[\begin{array}{c}
\rho u_{2} \\
\rho u_{1} u_{2} \\
p+\rho u_{2}^{2} \\
(e+p) u_{2}
\end{array}\right]
$$

Next we split Eqn. (66) as

$$
\frac{\partial U}{\partial t}+\frac{\partial}{\partial x}\left(G X^{+}\right)+\frac{\partial}{\partial x}\left(G X^{-}\right)+\frac{\partial}{\partial y}\left(G Y^{+}\right)+\frac{\partial}{\partial y}\left(G Y^{-}\right)=0
$$

where $G X^{ \pm}, G Y^{ \pm}$are split flux vectors given by

$$
G X^{ \pm}=\left[\begin{array}{c}
\rho\left(u_{1} A_{1}^{ \pm} \pm B_{1}\right) \\
\left(p+\rho u_{1}^{2}\right) A_{1}^{ \pm} \pm \rho u_{1} B_{1} \\
\rho u_{2}\left(u_{1} A_{1}^{ \pm} \pm B_{1}\right) \\
(e+p) u_{1} A_{1}^{ \pm} \pm\left(e+\frac{p}{2}\right) B_{1}
\end{array}\right], G Y^{ \pm}=\left[\begin{array}{c}
\rho\left(u_{2} A_{2}^{ \pm} \pm B_{2}\right) \\
\rho u_{1}\left(u_{2} A_{2}^{ \pm} \pm B_{2}\right) \\
\left(p+\rho u_{2}^{2}\right) A_{2}^{ \pm} \pm \rho u_{2} B_{2} \\
(e+p) u_{2} A_{2}^{ \pm} \pm\left(e+\frac{p}{2}\right) B_{2}
\end{array}\right]
$$

where

$$
\begin{aligned}
& \beta=\frac{1}{2 R T}, S_{1}=u_{1} \sqrt{\beta}, S_{2}=u_{2} \sqrt{\beta}, B_{1}=\frac{\exp \left(-S_{1}^{2}\right)}{2 \sqrt{\beta \pi}}, \\
& B_{2}=\frac{\exp \left(-S_{2}^{2}\right)}{2 \sqrt{\beta \pi}}, A_{1}^{ \pm}=\frac{\left(1 \pm \operatorname{erf}\left(S_{1}\right)\right)}{2}, A_{2}^{ \pm}=\frac{\left(1 \pm \operatorname{erf}\left(S_{2}\right)\right)}{2}
\end{aligned}
$$

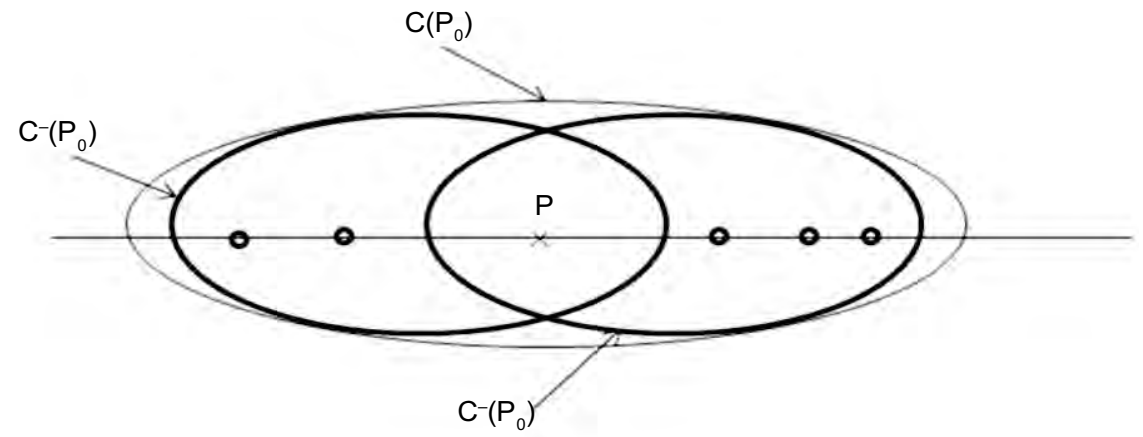

Figure 8. Left and right stencils of the node $P$ in one dimension. 
We now do stencil splitting as shown in Fig. 9(a) and Fig. 9(b).

The connectivity or stencil $C\left(P_{o}\right)$ of node $P_{o}$ is split in two ways, that is into $C_{1}\left(P_{o}\right), C_{2}\left(P_{o}\right)$ and $C_{3}\left(P_{o}^{o}\right), C_{4}\left(P_{o}\right)$ as shown in Fig. 9(a) and Fig. 9(b). The Eqn. (68) is then discretised as

$$
\left[\begin{array}{l}
\left(\frac{\partial U}{\partial t}\right)_{o}^{n}+\left(\frac{\delta}{\delta x} G X^{+}\right)_{C_{1}\left(P_{o}\right)}+\left(\frac{\delta}{\delta x} G X^{-}\right)_{C_{2}\left(P_{o}\right)} \\
+\left(\frac{\delta}{\delta y} G Y^{+}\right)_{C_{3}\left(P_{o}\right)}+\left(\frac{\delta}{\delta y} G Y^{-}\right)_{C_{4}\left(P_{o}\right)}
\end{array}\right]=0
$$

The discrete LS approximations used in Eqn. (70) are given by

$$
\left(\frac{\delta}{\delta x} G X^{+}\right)_{C_{1}\left(P_{o}\right)}=\left\{\frac{\sum \Delta y_{i}^{2} \sum \Delta x_{i} \Delta \tilde{G} X_{i}^{+}-\sum \Delta x_{i} \Delta y_{i} \sum \Delta y_{i} \Delta \tilde{G} X_{i}^{+}}{\sum \Delta x_{i}^{2} \sum \Delta y_{i}^{2}-\left(\sum \Delta x_{i} \Delta y_{i}\right)^{2}}\right\}_{i \in C_{1}\left(P_{o}\right)}
$$

$$
\left(\frac{\delta}{\delta x} G X^{-}\right)_{C_{2}\left(P_{o}\right)}=\left\{\frac{\sum \Delta y_{i}^{2} \sum \Delta x_{i} \Delta \tilde{G} X_{i}^{-}-\sum \Delta x_{i} \Delta y_{i} \sum \Delta y_{i} \Delta \tilde{G} X_{i}^{-}}{\sum \Delta x_{i}^{2} \sum \Delta y_{i}^{2}-\left(\sum \Delta x_{i} \Delta y_{i}\right)^{2}}\right\}_{i \in C_{2}\left(P_{o}\right)}
$$

$$
\left(\frac{\delta}{\delta x} G Y^{+}\right)_{C_{3}\left(P_{o}\right)}=\left\{\frac{\sum \Delta x_{i}^{2} \sum \Delta y_{i} \Delta \tilde{G} Y_{i}^{+}-\sum \Delta x_{i} \Delta y_{i} \sum \Delta x_{i} \Delta \tilde{G} Y_{i}^{+}}{\sum \Delta x_{i}^{2} \sum \Delta y_{i}^{2}-\left(\sum \Delta x_{i} \Delta y_{i}\right)^{2}}\right\}_{i \in C_{3}\left(P_{o}\right)}
$$

$$
\left(\frac{\delta}{\delta x} G Y^{-}\right)_{C_{4}\left(P_{o}\right)}=\left\{\frac{\sum \Delta x_{i}^{2} \sum \Delta y_{i} \Delta \tilde{G} Y_{i}^{-}-\sum \Delta x_{i} \Delta y_{i} \sum \Delta x_{i} \Delta \tilde{G} Y_{i}^{-}}{\sum \Delta x_{i}^{2} \sum \Delta y_{i}^{2}-\left(\sum \Delta x_{i} \Delta y_{i}\right)^{2}}\right\}_{i \in C_{4}\left(P_{o}\right)}
$$

The modified differences $\Delta \widetilde{G} X_{i}^{ \pm}, \Delta \widetilde{G} Y_{i}^{ \pm}$are given by

$$
\begin{aligned}
\Delta \tilde{G} X_{i}^{ \pm} & =\Delta\left(G X_{i}^{ \pm}\right)-\frac{\Delta x_{i}}{2}\left[\frac{\partial}{\partial x}\left(G X^{ \pm}\right)_{i}-\frac{\partial}{\partial x}\left(G X^{ \pm}\right)_{o}\right] \\
& -\frac{\Delta y_{i}}{2}\left[\frac{\partial}{\partial y}\left(G X^{ \pm}\right)_{i}-\frac{\partial}{\partial y}\left(G X^{ \pm}\right)_{o}\right]
\end{aligned}
$$

$$
\begin{aligned}
\Delta \tilde{G} Y_{i}^{ \pm} & =\Delta\left(G Y_{i}^{ \pm}\right)-\frac{\Delta x_{i}}{2}\left[\frac{\partial}{\partial x}\left(G Y^{ \pm}\right)_{i}-\frac{\partial}{\partial x}\left(G Y^{ \pm}\right)_{o}\right] \\
& -\frac{\Delta y_{i}}{2}\left[\frac{\partial}{\partial y}\left(G Y^{ \pm}\right)_{i}-\frac{\partial}{\partial y}\left(G Y^{ \pm}\right)_{o}\right]
\end{aligned}
$$

It may be noted that a few inner iterations are required for second-order accuracy. The well known time marching methods RK2/RK4 can be used for the semi-discrete law Eqn. (70) to obtain time accurate LSKUM based state update.

\section{5. q-LSKUM METHOD}

The q-variables or entropy variables were introduced by Deshpande ${ }^{10}$ for casting Euler equations in symmetric hyperbolic form. These variables have been used by Dauhoo, Ghosh, Ramesh and Deshpande ${ }^{9}$ and by Deshpande, Anandhanarayanan, Praveen and Ramesh ${ }^{13}$. For 1-D problem, the $q$-variables are defined by

$$
q=\left[\begin{array}{c}
\ln \rho+\frac{\ln \beta}{\gamma-1}-\beta u^{2} \\
2 \beta u \\
-2 \beta
\end{array}\right]
$$

The q-vector is a unique function of $\mathrm{U}$-vector and vice versa. The variables $\rho, u, \beta$ are

$$
\beta=-\frac{q_{3}}{2}, u=\frac{q_{2}}{2 \beta}, \rho=\exp \left[q_{1}+\beta u^{2}-\frac{\ln \beta}{\gamma-1}\right]
$$

Given $U$ or $q$, we can construct a unique Maxwellian denoted by $F$ or $F(q)$.

Because of logarithmic dependence of $\mathrm{q}$ on $\rho, u, \beta$ the linear combination

$$
\tilde{q}=(1-\theta) q^{\prime}+\theta q^{\prime \prime}
$$

of two q-vectors lead to a multiplication of corresponding Maxwellians. Let $F^{\prime}$ and $F^{\prime \prime}$ be the Maxwellians corresponding to $q^{\prime}$ and $q^{\prime \prime}$ and further let $\tilde{F}$ be the

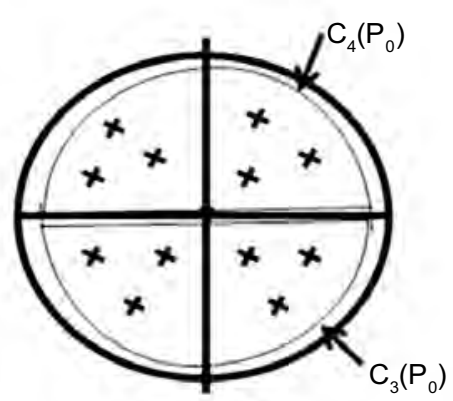

Figure 9. (a) Split stencil in $X$ direction and (b) Split stencil in $Y$ direction. 
Maxwellian corresponding to $\tilde{q}$. We then have $\mathrm{e}^{8,9}$

$$
\tilde{F}=\left(F^{\prime}\right)^{(1-\theta)}\left(F^{\prime \prime}\right)^{\theta}
$$

for which $\tilde{u}, \tilde{\rho}, \tilde{\beta}$ are given by

$$
\begin{aligned}
& \tilde{\beta}=(1-\theta) \beta^{\prime}+\theta \beta^{\prime \prime} \\
& \tilde{u}=\frac{(1-\theta) \beta^{\prime} u^{\prime}+\theta \beta^{\prime \prime} u^{\prime \prime}}{\tilde{\beta}} \\
& \tilde{\rho}=\left(\rho^{\prime}\right)^{(1-\theta)}\left(\rho^{\prime \prime}\right)^{\theta}\left[\frac{\left(\beta^{\prime}\right)^{(1-\theta)}\left(\beta^{\prime \prime}\right)^{\theta}}{\tilde{\beta}}\right]^{\frac{1}{\gamma-1}} \exp \left[-\theta(1-\theta)\left(u^{\prime}-u^{\prime \prime}\right)^{2} \frac{\beta^{\prime} \beta^{\prime \prime}}{\tilde{\beta}}\right]
\end{aligned}
$$

We want to obtain $\tilde{q}_{1}=\ln \tilde{\rho}+\frac{\ln \tilde{\beta}}{\gamma-1}-\tilde{\beta} \tilde{u}^{2}$ in terms of $q_{1}^{\prime}$ and $q_{1}^{\prime \prime}$.

The Eqn. (82) gives

$$
\begin{aligned}
\ln \tilde{\rho} & =\left[(1-\theta) \ln \rho^{\prime}+\theta \ln \rho^{\prime \prime}\right]+\frac{1}{\gamma-1}\left[(1-\theta) \ln \beta^{\prime}+\theta \ln \beta^{\prime \prime}\right] \\
& -\frac{1}{\gamma-1} \ln \tilde{\beta}-\theta(1-\theta)\left(u^{\prime}-u^{\prime \prime}\right)^{2} \frac{\beta^{\prime} \beta^{\prime \prime}}{\tilde{\beta}}
\end{aligned}
$$

The variable $\tilde{q}_{1}$ for the product of Maxwellian Eqn. (79) is then given by

$$
\begin{aligned}
& \tilde{q}_{1}=\ln \tilde{\rho}+\frac{\ln \tilde{\beta}}{\gamma-1}-\tilde{\beta} \tilde{u}^{2} \\
& =\left[(1-\theta) \ln \rho^{\prime}+\theta \ln \rho^{\prime \prime}\right]+\frac{1}{\gamma-1}\left[(1-\theta) \ln \beta^{\prime}+\theta \ln \beta^{\prime \prime}\right]- \\
& \theta(1-\theta)\left(u^{\prime}-u^{\prime \prime}\right) \frac{\beta^{\prime} \beta^{\prime \prime}}{\tilde{\beta}}-\tilde{\beta}\left[\frac{(1-\theta) \beta^{\prime} u^{\prime}+\theta \beta^{\prime \prime} u^{\prime \prime}}{\tilde{\beta}}\right]^{2} \\
& =(1-\theta)\left[\ln \rho^{\prime}+\frac{1}{\gamma-1} \ln \beta^{\prime}\right]+\theta\left[\ln \rho^{\prime \prime}+\frac{1}{\gamma-1} \ln \beta^{\prime \prime}\right]- \\
& \left\{\theta(1-\theta)\left(u^{\prime}-u^{\prime \prime}\right)^{2} \frac{\beta^{\prime} \beta^{\prime \prime}}{\tilde{\beta}}+\frac{\left[(1-\theta) \beta^{\prime} u^{\prime}+\theta \beta^{\prime \prime} u^{\prime \prime}\right]^{2}}{\tilde{\beta}}\right\}
\end{aligned}
$$

The sum in the curly brackets in Eqn. (83) can be simplified as follows:

$$
\begin{aligned}
& \text { Sum }=\frac{1}{\tilde{\beta}}\left[\begin{array}{l}
\theta(1-\theta)\left(u^{\prime}-u^{\prime \prime}\right)^{2} \beta^{\prime} \beta^{\prime \prime}+(1-\theta)^{2}\left(\beta^{\prime} u^{\prime}\right)^{2} \\
+\theta^{2}\left(\beta^{\prime \prime} u "\right)^{2}+2 \theta(1-\theta) \beta^{\prime} \beta^{\prime \prime} u^{\prime} u^{\prime \prime}
\end{array}\right] \\
& =\frac{1}{\tilde{\beta}}\left[\theta(1-\theta) \beta^{\prime} \beta^{\prime \prime}\left(u^{\prime 2}+u^{\prime \prime 2}\right)+(1-\theta)^{2}\left(\beta^{\prime} u^{\prime}\right)^{2}+\theta^{2}\left(\beta^{\prime \prime} u^{\prime \prime}\right)^{2}\right] \\
& =\frac{1}{\tilde{\beta}}\left[\begin{array}{l}
(1-\theta) \beta^{\prime}\left((1-\theta) \beta^{\prime} u^{\prime 2}+\theta \beta^{\prime \prime} u^{\prime 2}\right) \\
+\theta \beta "\left((1-\theta) \beta^{\prime} u^{\prime 2}+\theta \beta^{\prime \prime} u^{\prime 2}\right)
\end{array}\right] \\
& =(1-\theta) \beta^{\prime} u^{\prime 2}+\theta \beta " u{ }^{\prime 2}
\end{aligned}
$$

In view of these tedious algebraic manipulations, the Eqn. (83) simplifies to

$$
\begin{aligned}
\tilde{q}_{1} & =(1-\theta)\left[\ln \rho^{\prime}+\frac{\ln \beta^{\prime}}{\gamma-1}\right]+\theta\left[\ln \rho^{\prime \prime}+\frac{\ln \beta^{\prime \prime}}{\gamma-1}\right]-(1-\theta) \beta^{\prime} u^{\prime 2}-\theta \beta^{\prime \prime} u^{\prime 2} \\
& =(1-\theta)\left[\ln \rho^{\prime}+\frac{\ln \beta^{\prime}}{\gamma-1}-\beta^{\prime} u^{\prime 2}\right]+\theta\left[\ln \rho^{\prime \prime}+\frac{\ln \beta^{\prime \prime}}{\gamma-1}-\beta^{\prime \prime} u^{\prime \prime 2}\right] \\
& =(1-\theta) q^{\prime}+\theta q^{\prime \prime}
\end{aligned}
$$

We have thus shown that the addition of $q^{\prime}$ and $q^{\prime \prime}$ leads to multiplication of corresponding Maxwellians which itself is a Maxwellian $\tilde{F}$. This property of entropy variables has been used in constructing $q$-LSKUM method, i.e. the LSKUM method based on entropy variables ${ }^{9,13,30}$.

For developing q-LSKUM method, we first critically look at the LS formulae used in LSKUM method. For 1-D problems, we have employed the first-order accurate LS formula for the derivative $\left(\frac{\partial F}{\partial x}\right)_{x=x_{\mathrm{o}}}$.

$$
F_{x o}^{(1)}=\left(\frac{\sum_{i} \Delta x_{i} \Delta F_{i}}{\sum \Delta x_{i}^{2}}\right)_{i \in C\left(P_{o}\right)}
$$

and for second-order accurate derivative, we have defect correction formula

$$
F_{x o}^{(2)}=\left(\frac{\sum_{i} \Delta x_{i} \Delta \tilde{F}_{i}}{\sum \Delta x_{i}^{2}}\right)_{i \in C\left(P_{o}\right)}
$$

The two LS formulae look almost alike but there is an important difference. $\Delta \tilde{F}_{i}$ in the first-order accurate formula (85) is a difference between two Maxwellian distributions while $\Delta F_{i}$ in the second-order accurate formula is 'not' a difference between two Maxwellians. It is in fact given by

$$
\begin{aligned}
\Delta \tilde{F}_{i} & =\Delta F_{i}-\frac{\Delta x_{i}}{2}\left[\left(\frac{\partial F}{\partial x}\right)_{i}-\left(\frac{\partial F}{\partial x}\right)_{o}\right] \\
& =\left\{F_{i}-\frac{\Delta x_{i}}{2}\left(\frac{\partial F}{\partial x}\right)_{i}\right\}-\left\{F_{o}-\frac{\Delta x_{i}}{2}\left(\frac{\partial F}{\partial x}\right)_{o}\right\}
\end{aligned}
$$

It is a difference between two perturbed Maxwellians which strictly speaking is not a valid velocity distribution because it can become negative for some values of velocity $v$. Instead of the Maxwellians, now $q$-variables i.e., $q_{1}, q_{2}$ and $q_{3}$ was used. They have the following LS formulae for derivatives of $q$,

$$
q_{x o}^{(1)}=\left(\frac{\sum_{i} \Delta x_{i} \Delta q_{i}}{\sum \Delta x_{i}^{2}}\right)_{i \in C\left(P_{o}\right)}
$$




$$
q_{x o}^{(2)}=\left(\frac{\sum_{i} \Delta x_{i} \Delta \tilde{q}_{i}}{\sum \Delta x_{i}^{2}}\right)_{i \in C\left(P_{o}\right)}
$$

where

$$
\Delta \tilde{q}_{i}=\left[q_{i}-\frac{\Delta x_{i}}{2}\left(\frac{\partial q}{\partial x}\right)_{i}\right]-\left[q_{o}-\frac{\Delta x_{i}}{2}\left(\frac{\partial q}{\partial x}\right)_{o}\right]
$$

We define modified q-variables by

$$
\begin{aligned}
& \tilde{q}_{i}=q_{i}-\frac{\Delta x_{i}}{2}\left(\frac{\partial q}{\partial x}\right)_{i} \\
& \tilde{q}_{o}=q_{o}-\frac{\Delta x_{i}}{2}\left(\frac{\partial q}{\partial x}\right)_{0}
\end{aligned}
$$

The modified difference $\Delta \tilde{q}_{i}$ is then given by

$$
\Delta \tilde{q}_{i}=\tilde{q}_{i}-\tilde{q}_{o}
$$

It is noted that $\tilde{q}_{i}$ and $\tilde{q}_{o}$ are linear combinations of $q_{j}, j \in C\left(P_{o}\right)$. This is obvious when we note that the LS approximation of derivatives $\left(\frac{\partial q}{\partial x}\right)_{i},\left(\frac{\partial q}{\partial x}\right)_{o}$ are linear combinations of $q$ belonging $C\left(P_{i}\right)$ or $C\left(P_{o}\right)$. The modified $\tilde{q}_{i}$ and $\tilde{q}_{o}$ being linear combinations of $q$ they will have corresponding Maxwellians $F\left(\tilde{q}_{i}\right)$ and $F\left(\tilde{q}_{o}\right)$. In terms of these modified Maxwellians, we have second-order accurate LS formula

$$
F_{x o}^{(2)}=\left[\frac{\sum_{i} \Delta x_{i}\left\{F\left(\tilde{q}_{i}\right)-F\left(\tilde{q}_{o}\right)\right\}}{\sum \Delta x_{i}^{2}}\right]_{i \in C\left(P_{o}\right)}
$$

This second-order accurate LS formula has the same form as the first-order LS formula Eqn. (85). The first-order accurate numerical schemes are known to give smooth, wiggle free contours of $\rho, p, T$, have residue fall of several decades but smear the solution to unacceptable level. It is reasonable to expect that the above second-order accurate formula based numerical scheme will retain good properties of first-order scheme, still giving accurate solution with crisp flow features. This expectation is indeed supported by many computations done using $q$-LSKUM method $^{2,6,27,29}$.

\section{CONSERVATION PROPERTY OF LSKUM}

Questions about conservative nature of mesh-free numerical method are often raised. Following the celebrated paper by $\mathrm{Lax}^{19}$ on conservative numerical schemes, it is assumed that conservative schemes are essential for accurate capture of jump conditions. By implication, it is assumed that non-conservative numerical schemes will not yield accurate jumps such as for example Rankine-Hugoniot (RH) jump relations across a shock. They have computed many flows (subsonic, transonic, supersonic, hypersonic, and strongly rotating viscous flows) over years ${ }^{1,-3,6,8,27-30}$ using LSKUM method and always got accurate solution of Euler equations. There is a strong body empirical evidence to believe that LSKUM method, even though its conservative nature cannot be proved in the sense of finite volume method, always yields accurate flow solution. Recently, Katz and Jameson ${ }^{17}$ have also noted good agreement of mesh-free computations with conventional finite volume computations despite absence of any proof of conservation. Sridar and Balakrishnan ${ }^{31}$ gave experimental demonstration of mass conservation with mesh-free method. The issue is studied in more details.

They note that for every point or node $P_{i} \in$ cloud $\Omega$ of points, they generate connectivity $C\left(P_{i}\right)$. The LS formulae for discrete approximations to $F_{x o}, F_{y o}$ operate on the connectivity sets $C\left(P_{i}\right), P_{i} \in \Omega$. Also, set of connectivities form an 'overlapping cover' for $\Omega$. That is,

$$
\Omega=C\left(P_{1}\right) \cup C\left(P_{2}\right) \cup C\left(P_{3}\right) \ldots . . \cup C\left(P_{J}\right)
$$

Compare this situation with a finite volume method for which the triangulation $T_{i}$ provides a mutually disjoint cover, that is $T_{i} \cap T_{j}=$ either null, or an edge or a point. FVM achieves conservation property by having pairwise cancellation of fluxes at common edge between two triangles. This is not the case with mesh-free method LSKUM. Hence conservation property of LSKUM cannot be proven by proceeding on lines similar to the proof of conservation for FVM. 1-D LSKUM method is studied in more detail. The 1-D split Boltzmann equation

$$
\frac{\partial F}{\partial t}+\frac{v+|v|}{2} \frac{\partial F}{\partial x}+\frac{v-|v|}{2} \frac{\partial F}{\partial x}=0
$$

is discretised as

$$
\left[\begin{array}{c}
\frac{\partial F}{\partial t}+\frac{v+|v|}{2}\left(\frac{\sum_{i} \Delta x_{i} \Delta F_{i}}{\sum_{i} \Delta x_{i}^{2}}\right)_{i \in C^{+}\left(P_{\circ}\right)} \\
+\frac{v-|v|}{2}\left(\frac{\sum_{i} \Delta x_{i} \Delta F_{i}}{\sum_{i} \Delta x_{i}^{2}}\right)_{i \in C^{-}\left(P_{\circ}\right)}
\end{array}\right]=0
$$

Using Taylor series for $\Delta F_{i}$, one gets

$$
\begin{aligned}
& \left(\frac{\sum_{i} \Delta x_{i} \Delta F_{i}}{\sum_{i} \Delta x_{i}^{2}}\right)_{i \in C^{+}\left(P_{o}\right)}=\left(\frac{\sum \Delta x_{i}\left\{F_{x o} \Delta x_{i}+F_{x x o} \frac{\sum \Delta x_{i}^{2}}{2}+\ldots \ldots . . .\right.}{\sum \Delta x_{i}^{2}}\right)_{i \in C^{+}\left(P_{o}\right)} \\
& =F_{x o}+\left(\frac{\sum_{i} \frac{\Delta x_{i}^{3}}{2}}{\sum_{i} \Delta x_{i}^{2}}\right)_{i \in C^{+}\left(P_{o}\right)} F_{x x o}+\text { H.O.T. }
\end{aligned}
$$

Similarly,

$$
\left(\frac{\sum_{i} \Delta x_{i} \Delta F_{i}}{\sum_{i} \Delta x_{i}^{2}}\right)_{i \in C^{-}\left(P_{o}\right)}=F_{x o}+\left(\frac{\sum_{i} \frac{\Delta x_{i}^{3}}{2}}{\sum_{i} \Delta x_{i}^{2}}\right)_{i \in C^{-}\left(P_{o}\right)} F_{x x o}+\text { H.O.T. }
$$


Hence Eqn. (96) gives the modified pde at Boltzmann level.

$$
\begin{gathered}
{\left[\begin{array}{c}
\frac{\partial F}{\partial t}+v \frac{\partial F}{\partial x}+\frac{v+|v|}{2} K_{1}\left(P_{\circ}\right) F_{x x o} \\
+\frac{v-|v|}{2} K_{2}\left(P_{\circ}\right) F_{x x o}+H . O . T
\end{array}\right]=0} \\
K_{1}\left(P_{o}\right)=\left(\frac{\sum_{i} \frac{\Delta x_{i}^{3}}{2}}{\sum_{i} \Delta x_{i}^{2}}\right)_{i \in C^{+}\left(P_{o}\right)} \\
\text { where, } K_{2}\left(P_{o}\right)=\left(\frac{\sum_{i} \frac{\Delta x_{i}^{3}}{2}}{\sum_{i} \Delta x_{i}^{2}}\right)_{i \in C^{+}\left(P_{o}\right)}
\end{gathered}
$$

Taking $\psi$ - moment of Eqn. (99), one obtains the modified pde at Euler level.

$$
\left(\frac{\partial U}{\partial t}\right)_{o}+\left(\frac{\partial G}{\partial x}\right)_{o}+K_{1}\left(P_{o}\right) \frac{\partial^{2}}{\partial x^{2}}\left(G^{+}\right)+K_{2}\left(P_{o}\right) \frac{\partial^{2}}{\partial x^{2}}\left(G^{-}\right)=0
$$

Consider a steady 1-D shock with $F=F_{1}$ for $x<x_{o}$ and $F=F_{2}$ for $x>x_{o}$. Obviously $\rho_{1}, u_{1}, T_{1}$ and $\rho_{2}, u_{2}, T_{2}$ are related by Rankine-Hugonoit(R-H) jump conditions. In steady-state, the pde will enforce

$$
\frac{\partial}{\partial x}\left[G+K_{1}\left(P_{o}\right) \frac{\partial}{\partial x} G^{+}+K_{2}\left(P_{o}\right) \frac{\partial}{\partial x} G^{-}\right]=0
$$

that is $G_{1}=G_{2}$ because all gradients such as $\frac{\partial G^{+}}{\partial x}, \frac{\partial G^{-}}{\partial x}$ will be zero for uniform states 1 and 2. For shock propagating at constant velocity $s$, we will get

$$
G_{2}-G_{1}=s\left(u_{2}-u_{1}\right)
$$

Thus the LSKUM method satisfying the pde (in the limit of number of points $\rightarrow \infty$ ) will automatically ensure correct jump conditions across shocks. It is therefore not surprising that LSKUM method captures shocks quite accurately satisfying R-H jump conditions. Depending on the numerical diffusion present in the numerical scheme, the shock will be smeared. Smearing can be reduced by refining the cloud. The above arguments support the strong empirical basis for the claim that LSKUM method yields accurate flow solution capturing all flow features. We therefore believe that the conservation property is sufficient but not a necessary condition for getting accurate jump conditions across shocks.

\section{BOUNDARY CONDITIONS}

The boundary conditions have been derived from the concepts of kinetic theory of gases. Boundary conditions are theoretically introduced at the kinetic level and then the state update at Euler level is arrived at by using moment method strategy.

\subsection{Inviscid Wall Boundary Condition - Kinetic Characteristic Boundary Condition}

Kinetic Characteristic Boundary Condition (KCBC) was developed by Mandal ${ }^{22}$ to treat wall boundary condition. The wall boundary condition for inviscid flow states that the normal velocity of fluid is zero. This is called flow tangency boundary condition. This boundary condition is enforced using specular reflection model of kinetic theory of gases. In this model a particle incident on a solid wall has its normal velocity reversed after reflection from the wall. The tangential velocity remains unchanged. The velocity of reflected particle can be expressed in terms of velocity of incident particle as

$$
\vec{v}_{R}=\vec{v}_{I}-2\left(\vec{v}_{I} \cdot \hat{n}\right) \hat{n}
$$

where, $\vec{v}_{R}$ is the velocity of reflected particle, $\vec{v}_{I}$ is the velocity of incident particle, $\hat{n}$ - unit outward vector normal to solid wall.

Consider a point ' $\mathrm{P}_{0}$ ' on the wall. Let $\left(X^{\prime}, Y^{\prime}\right)$ be the local frame (Fig. 10) with $X^{\prime}$ ' along tangent and $Y^{\prime}$ along normal to the solid wall at point $P_{0^{\circ}}(X, Y)$ is the standard global frame. Further $\left(v_{1}^{\prime}, v_{2}^{\prime}\right)$ and $\left(v_{1}, v_{2}\right)$ are particle velocity components in respective frames.

The Maxwellian $F$ at $P_{0}$ is defined by,

$$
F= \begin{cases}F_{I}\left(v_{1}, v_{2}\right) & v_{2}^{\prime}<0 \\ F_{R}\left(v_{1}, v_{2}\right) & v_{2}^{\prime}>0\end{cases}
$$

$F_{I}$ and $F_{R}$ correspond to incident and reflected particles respectively. Using specular reflection model, the reflected Maxwellian $F_{R}$ can be expressed in terms of incident Maxwellian by,

$$
F_{R}=F_{I}\left(v_{1}-2 n_{x}(\vec{v} \cdot \hat{n}), v_{2}-2 n_{y}(\vec{v} \cdot \hat{n})\right)=F_{I}\left(v_{1}^{\prime},-v_{2}^{\prime}\right)
$$

$$
\text { for } v_{2}^{\prime}>0
$$

Here, $n_{x}, n_{y}$ are components of normal vector in the global frame. The update scheme for incident part at Boltzmann

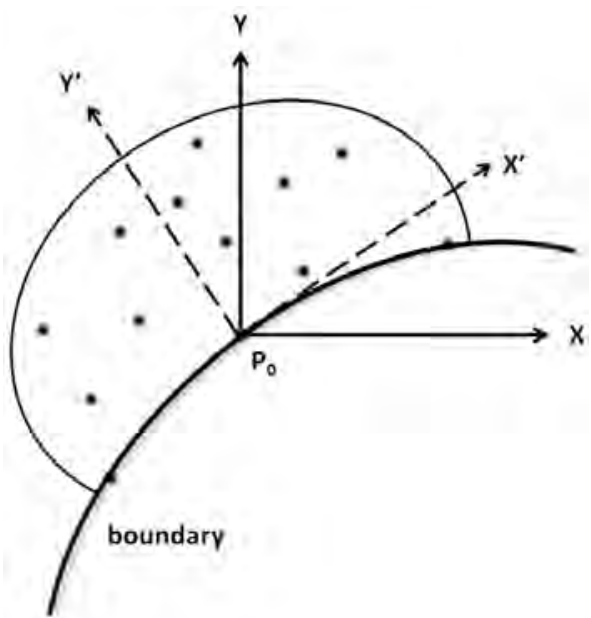

Figure 10. Local frame at a point on the wall. 
level is

$$
f_{I}^{n+1}=F_{0}^{n}-\Delta t\left[\left(v_{1}^{\prime} \frac{\delta F}{\delta x^{\prime}}\right)_{C_{1}\left(P_{o}\right)}+\left(v_{1}^{\prime} \frac{\delta F}{\delta x^{\prime}}\right)_{C 2\left(P_{o}\right)}+\left(v_{2}^{\prime} \frac{\delta F}{\delta y^{\prime}}\right)_{C_{4}\left(P_{o}\right)}\right]
$$

The discrete approximation $\frac{\delta F}{\delta x^{\prime}}$ and $\frac{\delta F}{\delta y^{\prime}}$ to spatial derivatives are defined by

$$
\begin{aligned}
& \frac{\delta F}{\delta x^{\prime}}=\frac{\sum \Delta y_{i}^{\prime 2} \sum \Delta x_{i}^{\prime} \Delta F_{i}-\sum \Delta x_{i}^{\prime} \Delta y_{i}^{\prime} \sum \Delta y_{i}^{\prime} \Delta F_{i}}{\sum \Delta x_{i}^{\prime 2} \sum \Delta y_{i}^{\prime 2}-\left(\sum \Delta x_{i}^{\prime} \Delta y_{i}^{\prime}\right)^{2}} \\
& \frac{\delta F}{\delta y^{\prime}}=\frac{\sum \Delta x_{i}^{\prime 2} \sum \Delta y_{i}^{\prime} \Delta F_{i}-\sum \Delta x_{i}^{\prime} \Delta y_{i}^{\prime} \sum \Delta x_{i}^{\prime} \Delta F_{i}}{\sum \Delta x_{i}^{\prime 2} \sum \Delta y_{i}^{\prime 2}-\left(\sum \Delta x_{i}^{\prime} \Delta y_{i}^{\prime}\right)^{2}}
\end{aligned}
$$

over substencils defined by

$$
\begin{aligned}
& C_{1}\left(P_{o}\right)=\left\{P_{i}, \forall i \in C\left(P_{0}\right) \mid x_{i}^{\prime}-x_{o}^{\prime}<0\right\} \\
& C_{2}\left(P_{o}\right)=\left\{P_{i}, \forall i \in C\left(P_{0}\right) \mid x_{i}^{\prime}-x_{o}^{\prime}>0\right\} \\
& C_{4}\left(P_{o}\right)=\left\{P_{i}, \forall i \in C\left(P_{0}\right) \mid y_{i}^{\prime}-y_{o}^{\prime}>0\right\}
\end{aligned}
$$

The reflected part $f_{R}$ can be obtained from specular reflection model and is given by

$$
f_{R}^{n+1}=f_{I}^{n+1}\left(v_{1}^{\prime},-v_{2}^{\prime}\right)
$$

Using the above definitions and taking $\Psi$ moments with suitable limits on integration we obtains the state update for $U_{P_{0}}^{\prime}$

$$
U_{P_{0}}^{\prime(n+1)}=U_{P_{0}}^{\prime(n)}-2 \Delta t\left[\begin{array}{l}
\left(\frac{\delta}{\delta x^{\prime}} G X^{\prime+-}\right)_{C_{1}\left(P_{o}\right)}+\left(\frac{\delta}{\delta x^{\prime}} G X^{\prime--}\right)_{C 2\left(P_{o}\right)} \\
+\left(\frac{\delta}{\delta y^{\prime}} G Y^{\prime-}\right)_{C_{4}\left(P_{o}\right)}
\end{array}\right]
$$

where

$$
\begin{aligned}
& G X^{\prime^{+-}}=\int_{0}^{\infty} \int_{-\infty}^{0} \int_{0}^{\infty} v_{1}^{\prime} \psi F_{I} d v_{1}^{\prime} d v_{2}^{\prime} d I \\
& G X^{\prime^{--}}=\int_{0}^{\infty} \int_{-\infty}^{0} \int_{-\infty}^{0} v_{1}^{\prime} \psi F_{I} d v_{1}^{\prime} d v_{2}^{\prime} d I \\
& G Y^{\prime-}=\int_{0}^{\infty} \int_{-\infty}^{0} \int_{-\infty}^{\infty} v_{2}^{\prime} \psi F_{I} d v_{1}^{\prime} d v_{2}^{\prime} d I
\end{aligned}
$$

\subsection{Far-field Boundary Condition - Kinetic Outer Boundary Condition}

Kinetic outer boundary condition (KOBC) was developed by Ramesh ${ }^{29}$ to treat the far-field boundary. The far-field boundary condition is derived in a similar fashion as the wall boundary condition. The state update at any point ' $\mathrm{P}_{0}$ ' on the outer boundary (Fig.11) is influenced by information going out of computational domain and information coming into the domain from free stream conditions. The outgoing

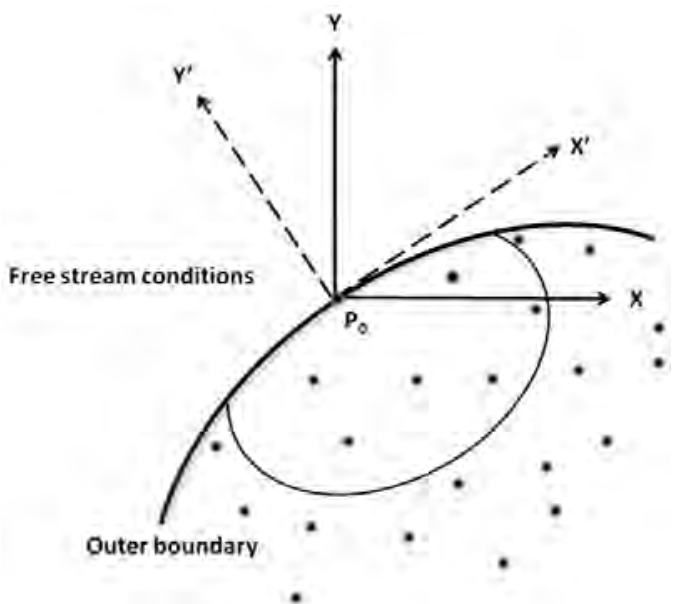

Figure 11. Neighbourhood stencil around a far-field boundary point.

information corresponds to velocity space with $v_{2}^{\prime}>0$ and incoming information corresponds to $v_{2}^{\prime}<0$.

Correspondingly the Maxwellian $F$ can be written as

$$
F=\left\{\begin{array}{cc}
F_{\text {in }}\left(v_{1}^{\prime}, v_{2}^{\prime}\right) & v_{2}^{\prime}<0 \\
F_{\text {out }}\left(v^{\prime}{ }_{1}, v_{2}^{\prime}\right) & v_{2}^{\prime}>0
\end{array}\right.
$$

where

$$
\begin{aligned}
& F_{\text {in }}=F_{\infty} \\
& f_{\text {out }}^{n+1}=F_{0}^{n}-\Delta t\left[\left(v_{1}^{\prime} \frac{\delta F}{\delta x^{\prime}}\right)_{C_{1}\left(P_{o}\right)}+\left(v_{1}^{\prime} \frac{\delta F}{\delta x^{\prime}}\right)_{C 2\left(P_{o}\right)}+\left(v_{2}^{\prime} \frac{\delta F}{\delta y^{\prime}}\right)_{C_{3}\left(P_{o}\right)}\right]
\end{aligned}
$$

for $v_{2}^{\prime}>0$

The spatial derivatives $\frac{\delta F}{\delta x^{\prime}}$ and $\frac{\delta F}{\delta y^{\prime}}$ are defined by Eqns (108) \& (109) and substencil $\mathrm{C}_{3}$ is defined by,

$$
C_{3}\left(P_{o}\right)=\left\{P_{i}, \forall i \in C\left(P_{0}\right) \mid y_{i}^{\prime}-y_{o}^{\prime}<0\right\}
$$

Now taking $\Psi$ moments with suitable limits on integration we gets (note that $\mathrm{F}$ corresponding to free-stream remains unchanged, i.e., free-stream condition),

$$
U_{P_{\circ}^{\prime}}^{(n+1)}=\bar{U}_{P_{\circ}^{\prime}}^{(n)}-\Delta t\left[\begin{array}{l}
\left(\frac{\delta}{\delta x^{\prime}} G X^{\prime+-}\right)_{C_{1}\left(P_{o}\right)}+\left(\frac{\delta}{\delta x^{\prime}} G X^{\prime--}\right)_{C_{2}\left(P_{\circ}\right)} \\
+\left(\frac{\delta}{\delta y^{\prime}} G Y^{\prime+}\right)_{C_{3}\left(P_{\circ}\right)}
\end{array}\right]
$$

The state vector $\bar{U}_{P}^{\prime}$ is defined as

$$
\bar{U}_{P_{\circ}}^{\prime}=\int_{0}^{\infty} \int_{-\infty}^{0} \int_{-\infty}^{\infty} \psi F_{\text {in }} d v_{1}^{\prime} d v_{2}^{\prime} d I+\int_{0}^{\infty} \int_{0}^{\infty} \int_{-\infty}^{\infty} \psi F_{\text {out }} d v_{1}^{\prime} d v_{2}^{\prime} d I
$$

\section{CONCLUSIONS}

The study has covered the theoretical developments in LSKUM, $q$-LSKUM, weighted LSKUM, defect correction for achieving higher-order accuracy, and treatment of boundary 
conditions by KCBC and KOBC. This development which began in 1989, is over a period of about twenty years and is due to the work of six PhD students at the Indian Institute of Science, Bangaluru. The present study does not cover R\&D work done in the development of LSKUMMN (LSKUM for moving nodes) method for computing unsteady flows involving multiple moving boundaries. The work has been supported by several research contracts from AR\&DB Delhi, ADA, Bangaluru, BARC, Mumbai, DRDL, Hyderabad and GTRE, Bangaluru. 2-D and 3-D $q$-LSKUM based codes have been developed by DRDL, Hyderabad and NAL, Bangaluru and these are routinely being used for computation of subsonic, transonic, supersonic and hypersonic flows past bodies at these laboratories.

\section{REFERENCES}

1. Mahindra, Ajit. Applications of least squares kinetic upwind method to strongly rotating viscous flows. Department of Aerospace Engineering, Indian Institute of Science, Bangaluru, 2004. MSc Thesis.

2. Anandhanarayanan, K. Development and application of a grid-free kinetic upwind solver to multibody configurations. Department of Aerospace Engineering, Indian Institute of Science, Bangaluru, 2003. PhD Thesis.

3. Anandhanarayanan, K.; Nagarathinam, M. \& Deshpande, S.M. Application of a grid-free kinetic upwind solver to a multibody configuration. AIAA Paper-2005-4846. 2005.

4. Arora, Konark \& Deshpane, S.M. Weighted least squares kinetic upwind method using eigenvector basis. Department of Aerospace Engineering, Indian Institute of Science, Bangaluru, FM Report No. 2004FM-17, 2004.

5. Arora, Konark; Rajan, N.K.S. \& Deshpande, S.M. Weighted least squares kinetic upwind method using eigenvector basis. In Eighth Annual CFD Symposium, CFD Division, AeSI, Bangaluru, 11-13 August 2005.

6. Arora, Konark. Weighted least squares kinetic upwind method using eigendirections (WLSKUM-ED). Department of Aerospace Engineering, Indian Institute of Science, Bangaluru, 2007. PhD Thesis.

7. Arora, Konark; Rajan, N.K.S. \& Deshpande, S.M. Weighted least squares kinetic upwind method (WLSKUM) for calculation of flow through blade passage using kinetic periodic boundary condition (KPBC). In Synposium for Advances in Aerodynamics and Design of Aerospace vehicles (SAROD-2005), 9-10 December 2005, Hyderabad, India..

8. Dauhoo, M.Z. The least squares kinetic upwind method based on entropy variables and its application to the understanding of the aerodynamics of lift. Department of Mathematics, University of Mauritius. PhD Thesis

9. Dauhoo, M.Z.; Ghosh, A.K.; Ramesh, V. \& Deshpande, S.M. q-LSKUM - A new higher order kinetic upwind method for euler equations using entropy variables. In Proceedings of Eighth International Symposium on Computational Fluid Dynamic (ISCFD), Bremen, Germany, September 5-10, 1999.
10. Deshpande, S.M. On the Maxwellian distribution, symmetric form and entropy conservation for the Euler equations. NASA Report No. NASA-TP-2583. 1986.

11. Deshpande, S.M. A second order accurate kinetic theory based method for inviscid compressible flows. NASA Report No. NASA-TP-2613. 1986.

12. Deshpande, S.M.; Mandal, J.C. \& Ghosh, A.K. Least squares weak upwind method for Euler equations. Department of Aerospace Engineering, Indian Institute of Science, Bangaluru. FM Report No. 1989-FM-4. 1989.

13. Deshpande, S.M.; Anandhanarayan, K.; Praveen, C. \& Ramesh, V. Theory and application of 3-D LSKUMbased on entropy variables. Int. J. Num. Meth. Fluids, 2001, 40(1-2), Septmber, 2002, 47-62.

14. Deshpande, S.M. Meshless method, accuracy, symmetry breaking, upwinding and LSKUM. Department of Aerospace Engineering, Indian Institute of Science, Bangaluru. FM Report No. 2003-FM-1. 2003.

15. Ghosh, A.K. \& Deshpande, S.M. Least squares kinetic upwind method for inviscid compressible flows. AIAA Paper-1995-1735. 1995.

16. Ghosh, A.K. Robust least squares kinetic upwind method for inviscid compressible flows. Department of Aerospace Engineering, Indian Institute of Science, Bangaluru. 1996, PhD Thesis.

17. Katz, A. \& Jameson, A. Edge based meshless methods for compressible flow simulations. In $46^{\text {th }}$ AIAA Aerospace Sciences Meeting and Exhibit, Reno, Nevada, 7-10 January 2008. AIAA Paper-2008-699.

18. Kuhnert, J. An upwind finite pointset method FPM for compressible Euler and Navier-Stokes equations. In Meshfree methods for partial differential equations, edited by M. Griebel \& M.A. Schweitzer. Springer LNCSE, 2002,

19. Lax, P.D. Weak solutions of non linear hyperbolic equations and their numerical computation. Comm. Pure Appl. Math., 1954, 7, 159-93.

20. Liszka T. \& Orkisz J. The finite difference method at arbitrary irregular grids and its application in applied mechanics. Computers and Structures, 1979, 11, 8395.

21. Lele, S.K. Compact finite difference schemes with spectral like resolution. J. Comp. Phy., 1992, 103 (1), 16-42.

22. Mandal, J.C. Kinetic upwind method for inviscid compressible flows. Department of Aerospace Engineering, Indian Institute of Science, Bangaluru, 1996. PhD Thesis.

23. Mandal, J.C. \& Deshpande, S.M. Kinetic flux vector splitting method for Euler equations. Computers and Fluids, 23(2), 447-78.

24. Malagi, Keshav. Rotationally invariant kinetic upwind method (KUMARI). Department of Aerospace Engineering, Indian Institute of Science, Bangaluru, 2006. MSc Thesis.

25. Malagi, Keshav S.; Kulkarni, P.S. \& Deshpande, S.M. KUMARI-kinetic upwind method with rotational 
invariance. In Proceedings of $11^{\text {th }}$ Asian Congress of Fluid Mechanics, Kuala Lumpur, Malayasia, May 2006, A37, pp 326-31.

26. Somasekhar, M.; Vivek, S.; Malagi, Keshav S.; Ramesh, V. and Deshpande, S.M. Adaptive cloud refinement ACR - Adaptation in meshless framework. In Eighth Asian Computational Fluid Dynamics Conference, Hong Kong, 10-14 January, 2010.

27. Praveen, C. Development and application of kinetic meshless methods for Euler equations. Department of Aerospace Engineering, Indian Institute of Science, Bangaluru, 2004. PhD Thesis.

28. Praveen, C. \& Deshpande, S.M. Kinetic meshless methods for compressible flows. Int. J. Num. Methods Fluids, 2007, 55(11), 1059-89.

29. Ramesh, V. Least squares grid-free kinetic upwind method. Department of Aerospace Engineering, Indian Institute of Science, Bangaluru, 2001. PhD Thesis.

30. Ramesh, V. \& Deshpande, S.M. Least squares kinetic upwind method on moving grids for unsteady Euler computations. Computers and Fluids Journal, 2001, 30, 621-41.

31. Sridar, M. \& Balakrishnan, N. An upwind finite difference scheme for meshless solvers. J. Comp. Phy., 2003, 189, 1-29.

32. Tiwari, S. \& Kunhert, J. A meshfree method for incompressible fluid flows with incorporated surface tension. Revue aurope'enne des elements finis, 2002, 11(7-8). Meshfree and Particle Based approach in Computational Mechanics. 2002.

\section{Contributors}

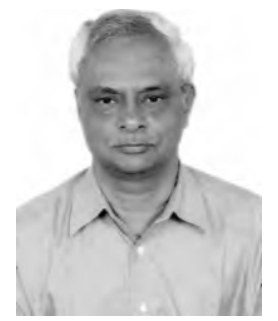

Prof S.M. Deshpande graduated in Electrical Engg from Nagpur University in 1963. He did his ME (Aeronautical Engineering) in 1965 and $\mathrm{PhD}$ in 1970 from Department of Aerospace Engineering, Indian Institute of Science, Bengaluru. Presently, he is working at the Jawaharlal Nehru Centre for Advanced Scientific
Research. His research interests are computational fluid dynamics, kinetic schemes, grid-free methods, computational aero-elasticity and data assimilation.

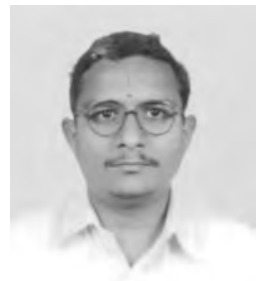

Dr Ramesh graduated in Mechanical Engineering from the University Visweswaraya College of Engineering, Bengaluru University in 1988. He did his Masters and $\mathrm{PhD}$ in Aerospace Engineering from the Indian Institute of Science (IISc), Bengaluru. He is working as a Scientist in the Computational and Theoretical Fluid Dynamcis Division of National Aerospace Laboratory (NAL) since 1991. His research interests are in the field of CFD, Aerodynamics, Grid-free methods, computational aero-elasticity

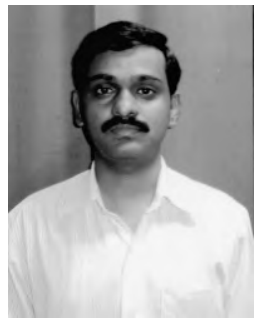

Mr Keshav S. Malagi graduated in Mechanical Engineering from Karnataka University, Dharwad in 2001. He did his ME from IISc, Bengaluru. He is working as a Scientist in the Computational and Theoretical Fluid Dynamcis Division of NAL since 2008. His research interests are computational fluid dynamics, aerodynamics, mesh-less methods, kinetic schemes, adaptation and computational aero-elasticity.

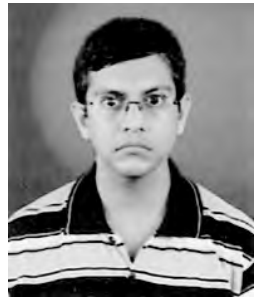

Mr Konark Arora obtained his BE (Hon) in Aeronautical Engineering from Panjab Engineering College (PEC), Chandigarh in 1998. He obtained his ME in Aerospace Engineering and $\mathrm{PhD}$ in Aerospace Engineering (CFD) from IISc, Bengaluru in 2001 and 2007 respectively. Currently, he is working in the CFD Division, DOCD, DRDL, Hyderabad. His areas of interest are computational fluid dynamics, aerodynamics, kinetic schemes and mesh-less methods. 